University of Rhode Island

DigitalCommons@URI

Open Access Master's Theses

1977

\title{
SYSTEMIC ABSORPTION OF TOPICALLY APPLIED SALICYLIC ACID
}

Bruce K. Birmingham

University of Rhode Island

Follow this and additional works at: https://digitalcommons.uri.edu/theses

\section{Recommended Citation}

Birmingham, Bruce K., "SYSTEMIC ABSORPTION OF TOPICALLY APPLIED SALICYLIC ACID" (1977). Open Access Master's Theses. Paper 181.

https://digitalcommons.uri.edu/theses/181

This Thesis is brought to you for free and open access by DigitalCommons@URI. It has been accepted for inclusion in Open Access Master's Theses by an authorized administrator of DigitalCommons@URI. For more information, please contact digitalcommons-group@uri.edu. 


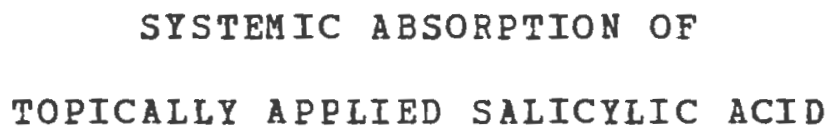




\section{MASTER OF SCIENCE THESIS \\ OF}

BROCE K. BIRIINGHAU

\section{Approved}

Thesis Comittee

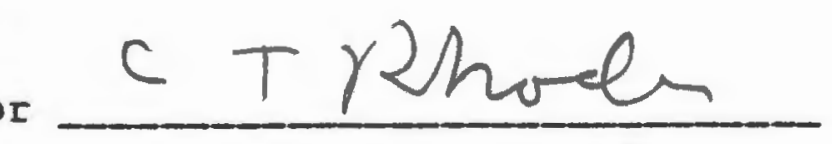
Major Professor
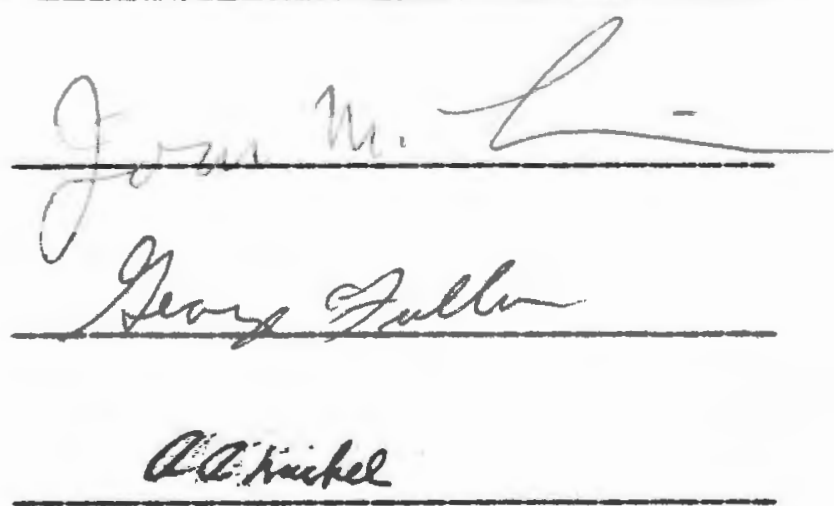

Dean of the Graduate School

UNIVERSITY OF RHODE ISLAND 


\section{ACKNOWL EDGEMENTS}

Acknowledgement is gratefully made to the faculty members of the college of Pharmacy of the university of Rhode Island for their suggestions and criticisms, and to Dr. James . Cooper, Jr. Eor his interest and enthusiasm. In particular, thanks are extended to Dr. George E. Osborne for his editorial efforts, and to Dr. Christopher T. Rhodes for his guidance and encouragement.

I would like to express special thanks to Dr. Douglas S. Greene for his advice and cooperation throughout this project. 


\section{ABSTRACT}

Salicylic acid was applied topically to the skin of rabbits and humans in order to study the pharmacokinetics of percutaneous absorption, and the effects of formulation and application variables on the rate and extent of absorption. Drug samples incorporated into hydrophilic ointment (USP) and hydrophilic ointment containing $10 \%$ urea, were applied to the shaved abdominal areas of female New Zealand rabbits for either four or eight hours. Drug samples used for the human studies were taken up in either hydrophilic ointment or in a polyethylene glycol/water solution. Blood samples vere drawn from the rabbits over a twenty-four hour period and from humans over an eight-hour period. Absorption of salicylic acid was determined by fluorometric analysis. Pharmacokinetic analysis of (blood) data resulted from two treatments: the ragner-Nelson methods; and a computerized, non-linear, least squares regression program NONIIN.

The systemic absorption of salicylic acid from hydrophilic ointment was substantial in rabbits; however. incorporation of urea, $10 \%(w / w)$ into the test ointment effected no significant change. The effect of ointment contact time on serum levels showed that the absorption process was essentially complete within six hours. The elimination curve for salicylic acid applied to animals fed 
during the test period exhibited an interesting and very substantial secondary peak not seen in fasted animals. The second peak may be due to biliary recycling.

The systemic absorption of salicylic acid from polyethylene glycol/water solution applied to human skin was negligible, suggesting the formation of a salicylate-glycol complex. Plasma concentrations resulting from the application of salicylic acid in hydrophylic ointment to the intact skin of humans were less than 1 mg. However absorption from the same system was increased considerably by disrupting the stratum corneum at the site of ointment application. 
Table of Contents

\section{Page}

$\begin{array}{lr}\text { Abstract } & i i \\ \text { List of Tables } & \nabla \\ \text { List of Figures } & \nabla i \\ \text { I. OBJECTIVES } & 1 \\ \text { II. INTRODOCTION } & 3 \\ \text { III. METHODS } & 25 \\ \text { IV. RESOLTS AND DISCOSSION } & 3 j \\ \text { V. CONCLOSIONS } & 6.5 \\ \text { VI. REFERENCES } & 68 \\ \text { VII. APPENDIX } & 73\end{array}$




\section{List of Tables}

Table

Page

I. Elimination Rate Constants

For Salicylic Acid Calculated

By Two Standard Methods

II. Half-Life of Salicylic Acid

In The Rabbit

III. Salicylic Acid Absorption Rate Constants Calculated By Two Standard Methods

IV. Absorption Lag Times For Topically Applied Salicylic Acid In The Rabbit

v. Areas Under The curve For Topically Applied Salicylic Acid In The Rabbit

VI. Pharmacokinetic parameters for Salicylic Acid obtained From The Percutaneous Application of Salicylic Acid In A Ruman Subject 
List of Figures

Figure

Page

I. Salicylic Acid concentration In The Rabbit Following The Topical Application of $10 \%$ Salicylic Acid ointment

II. Absorption Rate of Salicylic Acid

Calculated BY The Nagner-Nelson Method (Rabbit D)

III. Percent Salicylic Acid Remaining To Be absorbed (Rabbit E)

IV. Salicylic Acid Concentration (Rabbit J)

V. The Effect of Feeding On The Plasma Salicylic Acid Concentration Time Profile Following The Topical Administration of Salicylic Acid (Rabbit E)

VI. Plasma Salicylic Acid concentration In Human Pollowing The Percutaneous Application of $3 \%$ Salicylic Acid In $40 \%$ PEG/HATER

VII. Predicted Plasma Salicylic Acid Concentration In A Patient rith $30 \%$ Involvement of Total surface Area 


\section{OBJECTIVES}

Topical application of salicylic acid preparations is a well established practice in dermatology. of particular importance has been their use in the long term treatment of psoriasis. The literature is replete with reports of systemic salicylate toxicity, including fatalities, associated with the topical application of salicylic acid $(1-5)$. In these cases considerable quantities of drug were applied to large areas of skin, in which disease had

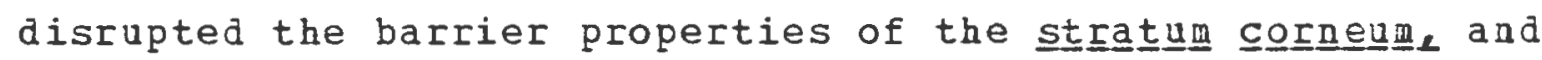
for which occlusive therapy was considered appropriate.

There is very little information available regarding either the rate or extent of systemic absorption of topically applied salicylic acid. Experimental procedures reported in the literature which deal with the percutaneous absorption of topically applied salicylic acid have not followed appropriate pharmacokinetic sampling methods; therefore, conclusions regarding bioavailability (i.e., the rate and extent of absorption) cannot be drawn.

Characterization of the pharmacokinetic parameters describing the systemic absorption of percutaneously applied salicylic acid is important for two reasons: first, it would provide information regarding the pharmacokinetics of a drug administered by a topical delivery system; and in addition, by characterizing the rate and extent of systemic absorption 
of salicylic acid, it may be possible to decrease the potential hazards faced by patients exposed to long term topical salicylate therapy.

The purpose of this project is therefore to study the systemic absorption of percutaneously applied salicylic acid, in rabbits as an animal model, and humans. The second objective is to examine the effect of formulation and application variables on the absorption of topically applied salicylic acid. Finally, it is planned to use salicylic acid to study the pharmacokinetcs of percutaneous absorption. 
II. INTRODUCTION

A. The Skin, Anatomy, and Physiology

skin, the largest organ of the body, consists of three anatomically distinct layers; the epidermis, the dermis, and the subcutaneous fat layer. The epidermis is a relatively thin, non-vascular, outer layer, which functions primarily as a barrier to the movement of material either into or out of the body. It may be subdivided into four layers, in ascending order; the stratuㅡㅁㅡ germaniti보므.

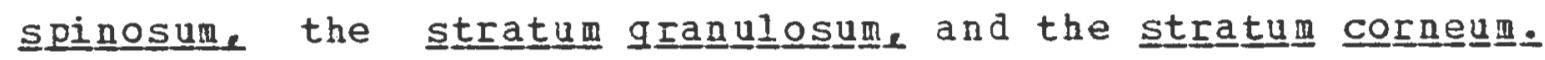
The cells of each layer are formed from those of the preceding layer beginning as the columnar, nucleated viable colls of the stratuuㅡ germanitiviunt and becoming the

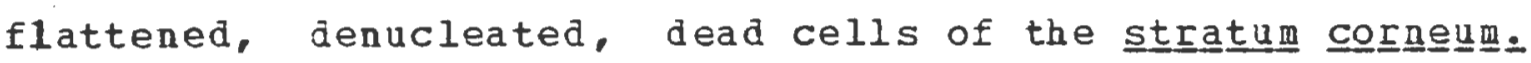
During this process the water content changes from about $70 \%$ to approximately 20, and the pH changes from about seven to four or five. In addition, keratin formation occurs from the cross-linking of poly-peptide chains by "peptide, and disulfide cysteine linkages", resulting in the hydrophobic, insoluble substance of the striatuuㅡ corngeun.

The epidermis is connected to the dermis by a series of conelike ridges or papillae. The dermis consists primarily of a network of densely packed, non-cellular, collagen and elastin fibers. It functions as a supportive systen for both blood and lymph vessels as well as for muscle and nerve 
fibers. In adaition, the dermis contains eccrine, sweat, and sebaceous glands and numerous hair follicles. It varies in thickness from three to five mo., has a pH of about 7.1 to 7.3 and contains $60 \%$ to $70 \%$ water.

Beneath the dermis lies the subcutaneous fat layer which may be of varied thickness, functioning as the insulation and shock absorber for the internal organs of the body.

several excellent revieys of the complete anatomy and physiology of the skin have been published $(6-8)$.

B. Barrier Punction of the straaum Corneum

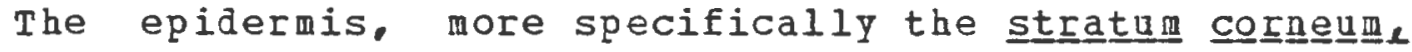
is generally recognized as the major barrier to the systemic absorption of drugs applied topically. Disruption of the integrity of the stratum corngeum has been shown to increase the diffusion of phenol and of strontium chloride $(9,10)$. Disease states such as exfoliative dermatitis and psoriasis increase the diffusion rates of sodium, zinc chloride, ammoniated mercury, and testosterone (11). The 1ow permeability of the stratum corneum compared to the viable layers of the epidermis $\left(10^{-9}\right.$ to $10^{-13} \mathrm{~cm}^{2} \mathrm{sec}^{-1}$ as compared to 10-6 $\left.\operatorname{cm}^{2} \sec ^{-1}\right)(12)$, characterize this layer as the principle barrier to diffusion. Through sequential stripping of the stratum corneuㅗㅛ with adhesive tape, Blank (13) showed that its barrier properties remained intact 
until the lowest layers were removed. Scheuplein (14) and Idson (15) have used these data and electron micrographs to characterize the entire stratuuㅡ corneum as a homogeneous barrier to penetration.

C. Routes of Percutaneous Absorption

1. Transepidermal absorption

Transepidermal absorption, which refers to absorption thtough the stratun corneum, may be subdivided into two categories, transcellular and intercellular. Transcellular diffusion represents the major route for the absorption of most substances.

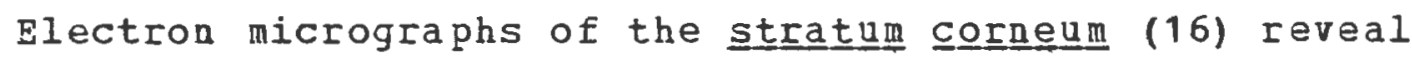
that keratin consists of distinct protein filaments surrounded by areas of lipid material so that separate lipid and polar regions exist within this layer. Lipid soluble and water soluble substances are thought to partition into and then diffuse through either the lipid or polar region depending on their relative affinities (14) creating two parallel routes for transcellular diffusion through the straauㅛ corneuㅡㅁㅡ.

Although the extent to which intercellular diffusion occurs has not been accurately determined, Scheuplein (17) cites the small intercellular volume available compared to that of the total stratum corneum electron micrographs 
showing extensive intercellular contact, and the existence of viscous, keratinous by-products within the intercellular spaces as factors limiting the importance of this route.

\section{Transappendageal Absorption}

Transappendageal absorption refers to the penetration of material through the eccrine and sveat glands and the pilo-sebaceous system, i.e., the sebaceous glands and hair follicles. The contribution made to the overall absorption process by these appendages is limited, since the area of skin they represent accounts for only about one percent of the skin's total surface area (18). For steady-state diffusion of most small, non-polar substances the role of transappendageal pathways is considered to be minimal. Before reaching the steady state, however, diffusion through

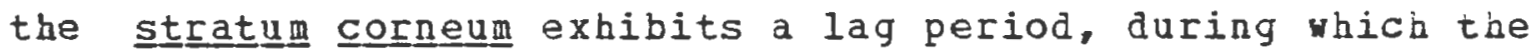
transappendageal route may offer an early "shunt" pathway $(6,7)$

Scheuplein et al. (19) has shown that for large polar molecules, (for example, steroids), transappendageal diffusion may offer the primary mechanism for systemic

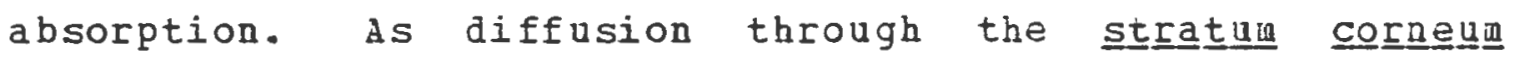
decreases, the importance of "shunt" diffusion increases, since the number of appendages ithin the area remains constant. As a result, the ratio of material diffusing through appendages to that passing transepidermally will 
increase.

D. Salicylic Acid

1. General Principles

Salicylic acid, ortho-hydroxybenzoic acid, has the chemical formula $\mathrm{C}_{7} \mathrm{H}_{6} \mathrm{O}_{3}$, and a molecular weight of 138,12 . It is found in the form of esters in several plants, notably in willow bark as salicin and in wintergreen as methyl salicylate. Today all salicylic acid is produced synthetically by heating sodium phenolate with carbon dioxide under pressure, a process developed by kolbe and Lautemann in the mid nineteenth century. (20)

Salicylic acid is available in either crystal or crystalline powder form. One gram dissolves in $460 \mathrm{ml}$. of water, $15 \mathrm{ml}$. of boiling water and $2.7 \mathrm{ml}$. of alcohol. The saturated aqueous solution has a pH of about 2.4 and the drug has a pkA of 3.3 .

2. Pharmacokinetics of Salicylic Acid

Following absorption, salicylic acid is rapidy distributed throughout body tissue and transcellular fluid. The highest concentrations are found in plasma, renal cortex, liver, beart, and lung. The lowest concentrations in the brain and skeletal muscle. It can be detected in 
synovial, spinal and peritoneal fluid, saliva and milk. salicylic acid is able to cross the placental barrier (21). Salicylic acid in plasma is $50 \%$ to $80 \%$ protein bound, largely to albamin (22). The volume of distribution varies with dose, at higher levels the proportion bound to plasia protein decreases and the apparent volume of distribution increases.

Salicylic acid is eliminated from the body by renal excretion of the unchanged drug or its metabolites. conjugation with glycine yields salicyluric acid (SD). conjugation with glucuronic acid gields salicylacyl glucuronide and salicyl phenolic glucuronide (SAG and SPG respectively). A fourth minor metabolite gentisic acid, (GA) is formed by oxidation (23).

The reported half-lives of salicylates in adults range from 2.4 hours at a therapeutic dose $(0.3$ grams per day) to nineteen hours at higher levels (greater than ten grams per day) (24). Levy has shown that an increasing half-life with increasing dose is due to saturation of the pathrays producing SO and SPG (25). The rates of formation of these metabolites may be described using Michaelis-yenton kinetics. Cumings and Martin (26) have calculated the threshold for saturation of su formation to be $276 \mathrm{mg}$. salicylic acid in the body. Below this level overall elimination appears to follow first order kinetics. At higher levels where so and SAG formation is limited, the overall elimination is occuring by parallel zero and first 
order processes. When very high levels of salicylic acid are present, SU and SPG formation accounts for a very small portion of total elimination and the overall process appears to be first order $(25,27)$.

Levy (23) has characterized the overall elimination of salicylic acid by the following equation in which elimination rate constants for $S O$ and $S A G$ are replaced by their appropriate Michaelis - Menton terms:

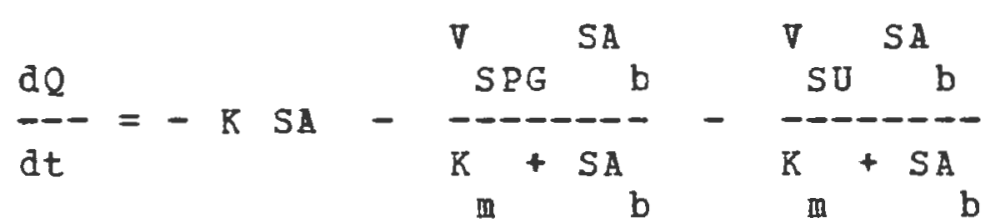

Equation (1)

Where

$k$ is equal to $k$ sa $+k$ sag $+k$ ga

$\mathbf{k}$ sa is the first order elimination rate constant of SA

k sag is the first order elimination rate constant of $G A$

$k$ ga is the first order elimination rate of $\mathrm{GA}$

$\nabla$ is the theoretical maximal rate of formation obtainable from either Michaelis-Menton or Iineweaver-Burke plots

SA body is the salicylic acid concentration in the body 
Z. Equations Governing Diffusion

Topically applied drugs penetrate the skin by passive diffusion. For conditions were the barrier provides the limiting step to diffusion, the steady state rate for diffusion may be described in terms of Fick's First Law;

$$
\frac{d Q}{d t}=-D A \quad \frac{d C}{d x}
$$

There

Equation (2)

dQ/dt is the steady state rate of diffusion

$D$ is the diffusion coefficient

expressed as the amount of material diffusing

per unit time per unit area,

A is the surface area

dc/dx describes an activity or concentration

gradient where $C$ is concentration and

$x$ is distance.

If $D$ is assumed constant for a given system of penetrant and membrane, and $A$ is constant then the driving force in passive diffusion is $d c / d x$, the concentration gradient.

An expanded form of equation (2) developed by Higuchi has been shown useful in characterizing the penetration of substances through the skin (28).

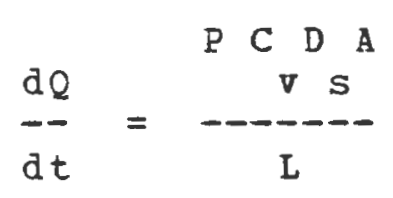

Where;

Equation (3)

DQ/DT is the steady state rate

of penetration 
$P$ is the effective partition coefficient

of drug between skin barrier and vehicle

$(P=C s / C V$, where; Cs is the concentration of

drug in the skin and $C \nabla$ is the concentration

of drug in the vehicle.

$C \nabla$ is the concentration of drug

dissolved in the vehicle

Ds is the diffusivity or average permeability

of the skin barrier

A is the area of skin to wich the

drug is applied

$\mathrm{L}$ is the effective thickness of the

skin barrier.

Equation 3 all on the physical ab chemical properties of the membrane, penetrant, and vehicle as described by $P$, the effective partition coefficient, and Ds, the diffusion constant of the drug in the ski: barrier. The partition coefficient bas been characterized by $\exists i g u c h i$ as the most important variable in determining diffusion rates, depending cn both molecular structure and particle size, while Ds varied only slightly for substances of similar molecular weight and shape.

These two terms have been combined to form a permeability coefficient, $\mathrm{kp}$, which specifies the permeability of a membrane to a given penetrant (29).

$$
k p=\frac{P D}{L}
$$$$
\text { Equation (4) }
$$

While equation three has been found useful in predicting steady-state diffusion rates it has been shown to 
have several shortccmings; the most serious involve the partition coefficient. This factor is generally determined in systems other than stratum corneum/vehicle, with olive oil/water being typical of the systems most commonly used. While there is evidence to show simple relationships between partition coefficients in one system compared to another, there are numerous deviations (30). Further, in defining tho partition coefficient as Cs/Cv, formulation variables are not considered. Higuchi (28) has suggested that $\mathrm{CV}$ be replaced by a thermodynamic activity term which incorporates properties of the vehicle into the partition coefficient and thus equation 3. The thermodynamic activity is defined as the concentration in the vehicle divided by the solubility in the vehicle ( $\mathrm{CV} / \mathrm{CS}$ ) and is a more accurate indicator of the driving force for diffusion. For a given system the thermodynamic activity of a penetrant in a vehicle and release rate from the vehicle increase up to saturation. There are a number of other limitations to the application of equation 3, for example, it applies only to steady-state diffusion rates in systems where the rate-limiting step is diffusion across the skin, and only for penetrants in low concentrations, in vehicle, since high concentrations cause deviations from Fick's Law.

Correlation of calculated diffusion rates with experimental results may be poor for several reasons. calculated rates depend on experiments in which the following parameters are generally controlled, i.e. (1) the 
concentration of penetrant in the vehicle remains constant, (2) sink conditions exist within the membrane, and (3) the characteristics of the vehicle remain constant (29). It is obvious that this type of control does not exist in the clinical setting. In addition, equation 3 does not account for the effect the vehicle or penetrant may have on the skin. The vehicle may cause hydration of the skin altering permeability, moreover, the penetrant may modify the barrier properties of the membrane or be bound within the skin, affecting the rate of penetration.

In spite of these limitations, the use of equation 3 and its derivatives is important in providing rough estimates of steady-state diffusion rates. 
F. Previous mork

Salicylic acid has been used by early workers attempting to characterize the mechanisms of percutaneous absorption and more recently by those interested in the effects of vehicles and/or additives on the rate of penetration. Barr (31) and Idson (32) have published excellent revieys of percutaneous absorption which refer to much of the earlier work in which salicylic acid was included. Notable among these early works are those of Kimura (33) and Nogami (34) who were among the first to consider the effect of the vehicle on the rate and extent of absorption of salicylic acid.

Much of the work dealing specifically with salicylic acid or salicylates has been published witinin the past twenty years and can be conveniently divided into in vit도으 and in $\underline{\text { vi }}$ vo studies.

\section{In Vitro}

Because of the limited application of in vitro results to in vivo situations, the number of significant studies is sma11. In vitro studies are essentially limited to determing penetrant release from ointment bases yithout characterizing absorption. Such systems for examing drag release typically rely on a "dialysis" or "diffusion" cell in which donor and receptor phases are separated by a 
membrane, usually cellophane or silicone rubber, although excised human skin has been used.

Howze and Billups (35) used a dialysis cell with a cellophane membrane to examine salicylic acid release from eight ointment bases. Release was determined by measuring salicylic acid concentration in the aqueous donor phase at various time intervals. Ranked from most rapid release to slowest, Howe and Billups reported oil/water emulsions greatest, followed by water/oil emulsions, oleaginous systems, and finally aqueous systems. A similar report by Billups and patel (36) corroborate the above pattern using a wider variety of individual ointment bases.

Nakano and patel (37) studied the in vitro release of salicylic acid from ointments using a dialysis spstem vith a silicone rubber membrane. The release rate reported was essentially the same as Howze and Billups (35) with the addition of polyethylene glycol ointment which showed the slowest release rate. Higuchi and Lach (38) have postulated the formation of a complex between salicylic acid and polyethylene glycol which may slow diffusion through the membrane.

In addition Nakano and Patel (37) examined the uptake of salicylic acid from ointment bases by reversing the donor and receptor phase and measuring the rate of uptake into the aqueous phase. The results show that in the absence of specific interactions between drug and base, the ointment that takes up salicylic acid the fastest, releases it the 
fastest.

Moisture and the extent of hydration are known to affect percutaneous absorption considerably. Mater, alcohol and dimethylsulfoxide were each incorporated into four ointment bases, wite petrolatum, cottonseed oil base, water-in-oil emulsion and an oil-in-water base. All three liquids enhanced release from white petrolatum and the water-in-oil base but inhibited diffusion from both the oil-in-water and cottonseed oil formulas. While no general explanations are possible, the results are important in showing that diffusion from ointment bases is influenced greatly by the inclusion of liguids.

Bottare 로 al. (40) studied the influence of drug concentration on the in 므보으 release of salicylic acid from ointment bases. In addition to reconfirming the release rate patterns established by previous workers, they applied the equation, developed by Kozumi and Higuchi (41) for those cases where diffusion through the vehicle is rate-limiting.

$$
\frac{d Q}{d t}=\sqrt{\frac{A D C_{S}}{2+}}
$$

Where;

Equation (5)

dQ/at is the rate of absorption

A is the concenetration of drug per unit volume.

$D$ is the diffusion coefficient constant

Cs is the drug solubility in the vehicle 
$t$ is time

The results indicate that release proceeds linearly only vithin the solubility range of drag within a given vehicle. Following saturation, increases in drug concentration show a smaller effect on release rates. Whether the change in release rates demonstrated is strictly due to saturation of the drug in a vehicle is questionable since the diffusion coefficient (D) has been shown to be concentration dependent in some cases (40). However, the correlation between release rates and concentration within a specific concentration range demonstrates the usefulness of this equation as a predictor of drug release rates.

\section{In iㅗ음}

Several workers have examined the effect of ointment bases on the release of salicylic acid in vivo. Early studies on the effects of various oily vehichles on the absorption of salicylic acid showed absorption from lard and lanolin but little from petrolatum (33). Strakosch, using emulsion bases, reported little effect of the vehicle on the absorption of salicylic acid (42). Several workers have since examined the effect of ointment bases on the release of salicylic acid in piro and shown a substantial effect due to the vehicle.

Stolar, BarI, and Rossi (43) have published york 
involving the percutaneous absorption of salicylic acid in which the primary aill was to develop a standardized procedure for determining absorption through intact skin. Ointments vere applied to intact shaved skin of rabbits by means of a bandage specifically designed to standardize the area of application. Absorption was characterized by determining the salicylic acid concentration in plasma at one hour intervals for a period of eight hours. The extent of absorption from each of four physical types of ointment bases, hydrophilic ointment, hydrophilic petrolatum. petrolatum, and polyethylene glycol ointment was determined. since hydrophilic ointment contains $37 \%$ water, the thermodyamic activity of salicylic acid in this vehicle would be expected to be high. On the basis of solubility and the anhydous nature of both bydrophilic petrolatum and petrolatum the thermodynamic activity of salicylic acid would predictably be lower. In polyethylene glycol the activity is lovest because of possible complex formation (38).

The results shown by stolar et al.e are in accord with predictions which can be made from equation 3 as modified by Higuchi (28). Colazzi (44) and Stelzer et al. (45) have also examined the effects of vehicle and shown similar patterns to those obtained by stolar et al.e Shelmire (46) working with water containing emulsions of salicylic acid, reported rates of penetration to be rapid, based on the thermodynamic activity of salicylic acid in aqueous 
vehicles.

Rashitake, et aㅡ. (47) compared absorption of salicylic acid from a series of oily vehicles by determing drug loss from the vehicle. The rate of loss yas found to be directly proportional to the oil partition coefficient of the drug. studies on the absorption of salicylic acid from polyethylene glycol 400 and four other hydrophilic vehicles shoved that the partition coefficient of the drug between the vehicle and benzene correlated well with the rate of absorption.

The effect of hydration on the absorption of salicylic acid has been studied by nurster and Kraemer (48). To avoid the influence of diffusion through vehicles, pure penetrants were used. Three liquid salicylate compounds were tested; ethyl salicylate, methyl salicylate and ethyleneglycol salicylate. Absorption was measured as a function of total salicylate recovered in urine. A hydrated condition was created by occlusion with a specially designed "absorption cell"; the anhydrous state was produced by incorporating a dessicant into the cell. A significant increase in the rate of absorption was caused by the hydrous condition of the skin. The increase was greatest for the most water soluble salicylate (ethyleneglycol salicylate). For the three esters studied, the extent of increase in absorption was shown to be inversely proportional to the oil/water partition coefficient and directly proportional to its aqueous solubility. 
Wurster and Kraemer (48) also studied the effect of defatting the skin on the absorption of salicylic acid. The skin was defatted by immersing the subject's forearm in ethyl ether for a one minute period. Methyl salicylate yas applied as previously described and arinary salicylate levels were determined. The same procedure was carried out on skin not treated with ether. Following the defatting procedure the absorption of methyl salicylate was $27 \%$ less than that observed in subjects who had not been treated vith ether. The authors suggest that the difference in absorption is primarily due to the destruction of the lipid pathways through which the drug normally diffuses. In adition, the ether extraction ma have directly removed water from the skin or alter the lipid membrane to create a partially dehydrated condition. The resulting anhyrous condition of the defatted skin would result in a more effective barrier to penetration than normal or bydrous skin.

Higuchi (28) has stated that the driving force for percutaneous diffusion is the thermozynamic activity or activity coefficient. He has also shown that for yeakly acidic drugs, such as salicylic acid, activity is inversely proportional to $10^{\mathrm{pH}}$. Arita et al. (49) and Marcus et al. (50) have examined the effects of $\mathrm{pH}$ on the rata and extent of absorption of salicylic acia.

Arita and colleaques, using the intact skin of guinea pigs, determined the absorption of salicylic acid as the 
percent of drug lost from Sorenson's phosphate buffer over a six hour time period. Varying the pH from an initial value of 2 to 5 caused a decrease in the amount of salicylic acia absorbed from six percent at pH 2 to essentially zero at a $\mathrm{pH}$ of 5. As the pH changed from 2 to 5 , the authors noted a decrease in the oil/water partition coeficient, pointing out the importance of this term in percutaneous absorption.

Marcus et a1. (50), examined the effect of $p H$ on the absorption of salicylic acid from hydrophilic ointment. Absorption was studied by measuring salicylate blood levels in rabbits according to the method of stolar et al. (43). At low pH (2.97) absorption was significantly higher than at intermediate levels, i.e., 4.48, 6.80, and 9.23. These results would be predictable from the work of Higuchi and Lach (38). Marcus et al. (50) noted that dissolution is often the rate limiting step in absorption from the gastrointestinal tract and may be a factor in this case.

The effect of additives on the absorption of drugs from various vehicles has long been of interest. Marcus et al. (50), Stelzer et al.s (45) and shen et al. (51) have studied the effects of formulation additives on the percutaneous absorption of salicylic acid.

Stelzer et al. (45) examined the influence of dimethyl sulfoxide on the absorption of salicylic acid in each of four ointment bases. Absorption was determined by the method of stolar et a1. (43). The addition of 15\% Daso to these bases and to a fourth base, polyoxyethylene staryl 
ether gel. produced mixed results. The absorption of salicylic acid from hydrophilic ointment and hydrophilic petrolatum was significantly increased. Little effect was shown on the absorption characteristics of either polyethylene glycol ointment or the ether gel. The authors report that the $10 \%$ salicylic acid was completly solubilized by the DMSO in all four ointment systems. It has been previously shown that organic solvents penetrate the skin and enhance the percutaneous absorption of drugs by solubilizing the lipoidal aterials of the cell walls. stelzer et al. (45) attribute the increase 2 absorption of salicylic acid to the ability of DMso to solubilize salicylic acid and then serve as a penetrant carrier. It should be pointed out however, that the precise mechanism by which DMso enhances permeability is essantially unknown (43). In the same study, DMSO had little effect on the absorption of the glycol ointment bases. The possibility of complex formation between glycols and salicylic acid has previously been reported (38). It is also known that drugs held within vehicles by such complexes exhibit Iow thermodynamic activites. The negligible effects of Duso on absorption from the glyccl bases in this study suggest that the penetrant has little effect on the complex and as such does not significant II improve the thermodynamic activity of salicylic acid in these vehicles.

Marcus et a1. (50) also showed an increase in the absorption of salicylic acid from hydrophylic ointment over 
a $\mathrm{p} \theta$ range from 2.97 to 10.78 following the addition of DHSO. However, its influence on the rate of absorption and on peak levels was less pronounced at the higher pH values. The authors suggest that this finding indicates that the effect of DMSO on absorption is a function of its ability to solubilize salicylic acid within the ointment base rather than any direct effect as a penetrant or carrier.

Shen and co-workers have recently examined the effects of non-ionic surfactants on percutaneous absorption of salicylic acid in the presence of DMSO (51). Ten percent salicylic acid was incorporated along with $10 \%$ DMso into wite petrolatum containing a variety of surfactants. The method for studying absorption was based on the work of stolar et al. (43). Results indicate that a wide range of non-ionic surfactants improved absorption of salicylic acid and sodium salicylate. The mechanism by which surfactants enhance absorption is unknown but a dimethylsulfoxide-drug-surfactant complex is suggested. Shen et $\underline{\text { l }}=$ (51) also suggest that the formation of such a complex results in an increased activity coefficient, accounting for an increase in absorption as predicted by Higuchi (28).

The effects of percutaneous absorption through daraged skin have been discussed previously. Stoughton (6), Katz (7). and hurster (52) have published excellent revieus on percutaneous absorption which include discussion on tae effects of removal of the barrier layer of the skin. 
Rashitake et al. (53) have examined the effect of repetative stripping of the stratum corneum on the percutaneous absorption of salicylic acid. Absorption vas calculated as the amount of salicylic acid remaining in the test vehicle over a six hour period. The results shoved a significant increase in the amount of arug absorbed: stripped skin exhibited a ten-fold increase compared to intact skin. The lag time required to reach steady state diffusion through intact skin was not determined.

In wat may be considered the first clinical pharmacokinetic study inrolving salicylic acid, taylor and Halprin (54) examined the absorption of salicylic acid in four patients suffering from psoriasis. Salicylic acid was applied in the form of a gel containing 6 percent drug in a base containing $60 \%$ propylene glycol and $19.4 \%$ alcohol. The gel was applied in a fixed amount to the entire body surface of the patients and the treated areas occluded with plastic wrap. The dressing was left intact for a period of ten hours. The procedure was repeated daily for five days. serum samples were collected at six hour intervals for the entire test period. When applied under occlusion, salicylic acid was rapidiy absorbed from the glycol and alcohol base, 60\% of the applied drug was absorbed. Serum salicylic acid levels aid not exceed $5 \mathrm{mg}$., well below the lower limit of what is considered a toxic level (30 mg) (55). 
III. METBOD

A. Materials

1. Chemicals

Salicylic Acid, A.C.S. (lot \#743715)'2

Sodium Tungstate A.C.S., (lot \#744002 \& 755089)1

Sodium Hyaroxide A.C.S. (1ot \#752642)1

Ferric Nitrate, A.C.S., (lot \# 751140)1

Mercaric Chloride, A.C.S., (lot \# 752538)1

Orea, A.C.S., (lot 751363) 1

Hydrophilic ointment, D.S.P., (lot \#Y4663k01)2 Bydrochloric Acid, A.C.S., (lot \#1090 216R60) 3 Sulfuric Acia, A.C.S., (lot \# E 911015) Polgethylen GIYcol 400, O.S.P., (Iot Y4023J23)* Liquamin Sodium "10" Sodium Heparin Injection, 0.S.P. (lot \#6853575811) 5

1. Fischer Scientific Company, Rairlawn, New Jersey

2. Armand Drug and Chemical Co., Irvington New Jersey

3. B E A, Allied Chemical Specialty Divisions, Morristown, New Jersey

- Ruger Chemical Co, Irvington, New Jersey

5. Organon Incorporated, Nest Orange, New Jersey 
B. Reagents

Tungstic Acid Reagent was prepared by mixing together ten percent aqueous sodium tungstate solution $(10 \mathrm{ml}$.$) and 1 / 12 \mathrm{~N}$ sulfuric acid $(80 \mathrm{ml}$.$) .$

Trinder's Reagent was prepared by dissolving, with heat, forty grams of mercuric chloride in approximately $700 \mathrm{ml}$. of distilied water. Upon cooling, $120 \mathrm{ml}$. of $1 \mathrm{~N} \mathrm{HCl}$ and forty grans of ferric nitrate were added, and the solution brought to one liter with distilled water. (The solution, when stored in a refrigerator, is reported stable for several months.)

C. Equipment and Supplies

Fluorometer, Turner Mode1 111, G.K. Turner Associates, palo Alto, California

Spectrophotometer, Perkin-Elmer, Model 200, Coleman Instruments Division, Oak Brook, Illinois

Ointment Mill, Hamonia Model, Josef Deckelmann, Aschaffenburg. West Germang

Butterfly-21, Intermittent Infusion set, Abbott Laboratories, North Chicago, Illinois

Plastipak Disposable $3 \mathrm{ml}$. Syringes, Becton-Dickinson and Company, Rutherford, New Jersey

Sterile, Disposable Needles, 25G, 5/8", Becton-Dickinson and Company, Rutherfood, New Jersey 
Vacutainer, Evacuated glass tubes, Beckton-Dickinson and Company, Rutherford, New Jersey

Rabbits, New Zealand Females, five to six pounds, Gloucester Rabbitry. Chepachet, Rhode Island

Glassware and common laboratory equipment as available in the College of Pharmacy 
D. Procedures

1. Ointment Preparation

Hydrophilic ointment U.S.P., obtained comercially, was milled, using a Hamonia ointment ill, to improve consistency and ease of application.

a. 10\% Salicylic Acid Ointment

To provide a more uniform particle size range for incorporation into the ointment base, salicylic acid, A.C.S., fine crystal, was passed through an 80-mesh seive. six grams of the salicylic acid were then incorporated into 54 grams of hyảrophylic ointment using the pharmaceutical technique of geometric dilution. No levigating or preservative agents were incorporated into the ointment base. Hard rubber spatulas were used throughout the procedure to aroid any possible contact between iron and salicylic acia wich produces the iron salicylate complex manifest by a purple coloration of the ointant. 
b. $10 \%$ Drea, 10\% Salicylic Acid Ointment

Six grams of urea, passed through an 80 mesh seive, were incorporated into 24 grams of previously milled hydrophilic ointment by the method of geometric dilution. By the same technique, six grams of salicylic acid were incorporated into 24 grams of hydrophylic ointment. The two ointment mixtures vere combined, geometrically, to produce a test ointment containing $10 \%$ urea and $10 \%$ salicylic acid $(w / w)$.

2. Rabbit Preparation

Female New zealand rabbits, age approximately five months, weighing two to three kilograms, were housed in humidity and temperature controlled quarters and maintained on a diet of Charles River Rabbit Chow and water a $\underline{a} \underline{\underline{I}} \underline{\underline{i}} \underline{\underline{b}}$ The animals vere observed for a period of one week following delivery; if no signs of ill health were detected the rabbits were released for experiments.

Clay and Nelson (56) have suggested that the dermal permeability of drugs may be altered by stress; therefore, all rabbits to be used for experimentation were put through an established routine. For several days prior to application of a test ointment, each rabbit was placed in a restraining cage for increasing periods of time, in order to condition the animal to the restraining box and relieve stress associated with this test condition. Two to three 
hours prior to application of a test ointment, the hair on the ventral side of the animal from the forelegs to the hindlegs was removed with animal clippers, taking care not to cut or otherwise visibly damage the skin.

Immediately prior to application of ointment, an intermittent infusion set, 21G, 5/8" needle, was inserted into the main ear artery. A blood sample was collected to serve as a zero time determination and the infusion line was cleared with a dilute (100 units/ml.) beparin solution to prevent clot formation.

An accurately weighed sample of ointment vas spread over a standard, rectangular, ( $7 \mathrm{~cm}$. $\mathrm{x} 13 \mathrm{~cm}$ ) template (Similar to the control technique reported by stolar et al. (43)). The template was centered on the adhesive surface of a strip of adhesive tape; the assembly was carefully inverted and applied to the previously shaved area of the animal. The rabbit was then placed in a restraining box for the duration of the experiment. Hater was provided ad lib.․ food was given or vithheld depending on the test conditions for the particular study. Blood samples were drawn at specified intervals for twenty four hours following initial application of the test ointment. All blood samples were allowed to clot, centrifuged at approximately 2000 BP for ten minutes, and then at least one ml. of serum was drawn off and stored under refrigeration until analysis.

Since the proper determination of the pharmacokinetics of a drug usually involves the collection of blood samples 
for a period of four to five half-lives following its administration, the characterization of the pharmacokinetics of percutaneously absorbed salicylic acid would likely require a sampling time of twenty to twenty four hours. The collection method used in this investigation was a modification of that described in the literature. Instead of hourly puncture of the ear vein, the previously described infusion set was placed in the ear artery and remained in place throughout the sampling period. Thus, blood could be drawn at any time; the insertion of only one needle considerably reduced the possibilities of infection or damage to the artery.

The catheter was kept filled with dilute heparin solution between sample collections to prevent obstructions from forming. Hoverer, obstruction of the catheter occasionally occured, particularly during the last six or eight hours of an experiment, and was generally due to the collapse of the artery around the needle. In some cases this was remedied by simply flushing the catheter with heparin, or gently warming the ear with a sunlamp. When in a few instances the obstruction persisted, the other ear was catheterized or the experiment vas terminated.

Collapse of an artery or damage to the vessel wall (e.g. scarring) often made it impossible to use a test animal for more than one experiment, even after a three or four week recovery period. Permanent catheterization of the carotia artery may provide a more reliable method of sample 
collection during the course of an experiment; it may also offer a method by which animals could be used for more than a single experiment.

\section{Human Preparation}

A detailed report of the proposed project, its objectives, methods, and potential hazards was submitted to both a University Humans Studies Committee and an Institutional Review Board for approval (Appendix). Following the guidelines established by these committees, volunteers were asked to refrain from taking ang aspirin or aspirin-containing products for one week, and all drugs, including alcohol, for 48 hours preceding an experiment. Prior to application of a test vehicle, an intermittent infusion set, 25G, 5/8" needle was inserted into a vein on the back of the subject's hand by a Registered Nurse and a blood sample drawn. The infusion line was then cleared with a dilute (100 unit/ml.) heparin solution to prevent clot formation between sampling times. The appropriate test vehicle was then applied to the subjects forearm, for the prescribed length of time, after which the skin surface was washed with warm water and soap

Two vehicles were used for human experiments; polyethylene glycol 400 and water, and $10 \%$ salicylic acid in hydrophylic ointment. Because one system is a liquid wile the other a solid, it was necessary to have different 
methods of application.

Fifteen liters of the test solution (PEG400:water) were placed in a ten gallon glass tank, constantly stirred and maintained at a temperature of 37 degrees. Each subject immersed his hand, forearm and distal portion of the upper arm in the solution for a period of two hours. For the solid system, ointment was applied to the subject's forearm and spread evenly over the entire skin surface. The forearm was then occluded with plastic film and the ointment allowed to remain in place for a period of two hours. The second test condition was a modification of the first in which the subject shared the hair off the forearm the night before the test. Immediately prior to application of the ointment the skin of the volunteer's forearm was "stripped" with adhesive tape five times. This process consisted of taping the forearm from wrist to elbow and then removing the tape along the entire forearm. The test conditions in this case required application of the ointment for three hours.

E. Methodology

1. Pluorometric

The primary assay used was the spectrofluorometric method developed by saltzman (57). The method makes use of the fluorescence of the salicylate ion on exposure to ultraviolet light. 
Nine and one-half ml. of tungstic acid reagent was added to $0.5 \mathrm{ml}$. of serum; the solution was shaken and allowed to stand at least ten minutes. The mixture was then filtered through medium retentive filter paper and the filtrate collected. Five ml. of filtrate were pipetted into a test tube and 7 ml. of $10 \mathrm{~N} \mathrm{NaOH}$ added. The solution was mixed, a portion transferred to a cuvette, and the fluorescence determined within thirty minutes in a Turner Fluorometer fitted with a primary filter (7-60) and secondary filters $(110-827$ and $110-823)$.

A standard reference curve was produced by adding 0.5 ml. of varying concentrations of salicylic acid to $4.5 \mathrm{ml}$. tungstic acid reagent. Seven ml. of $10 \mathrm{~N} N a O H$ was added, the solution was mixed, and the fluorescence determined within thirty minutes. A linear Beer's plot was obtained over tine concentration range of $0.5 \mathrm{mg} \%$ to $20 \mathrm{mg} \%\left(I^{2}=0.99\right)$.

In this procedure tungstic acid reagent serves to precipitate serum proteins and separate salicylic acid present. Sodium hydroxide serves a dual purpose, the first is to hydrolyze all salicylate metabolites present to salicylic acid. In adition, saltzman (57) has shown that an alkaline condition will increase the fluorescence of salicylates; maximum fluorescence was produced using $10 \mathrm{~N}$ $\mathrm{NaOH}$. 
2. Spectrophotometric

The secondary assay for the determination of salicylic acid in blood relies on the purple color given by a salicylate-ferric ion complex. The procedure used is that developed by Trinder (58), as modified by Biber and Rhodes (59).

Two ml. of serum was pipetted into a $10 \mathrm{ml}$. volumetric flask. Five ml. of Trinder's Reagent was then added. The $\mathrm{pH}$ was adjusted to 1.5 with $0.3 \mathrm{~N} \mathrm{NaOH}$ and the solution then brought to vo? $=$ with distilled water. A portion of the solution was trusserred to a quartz cuvette and the absorbance determined at a wavelength of $540 \mathrm{~nm}$.. using a model 200 Perkin-Elmer spectrophotometer.

The calibrat. in curve produced a Beer's plot linear over a salicylic acid concentration range of $5 \mathrm{mg} \%$ to $100 \mathrm{mg}$ ( $\mathrm{r}^{2}$ $=0.99)$.

In this procedure, mercuric chloride and hydrochloric acid are used to precipitate plasma proteins, ferric nitrate to generate the salicylic acid-ferric ion complex and the purple color on which the colorimetric analysis is based. 


\section{RESULTS AND DISCUSSION}

\section{A. Animal Pharmacokinetics}

1. Elimination Rate Constants

Elimination rate constants for salicylic acid were calculated for each treatment by each of two methods. First, when the common logarithm of serum salicylic acid concentration was plotted versus time, the slope of the terminal portion of the curve yielded the el. : ation rate constant, ke. A representative plot of sise log concentration versus time plot is shown in Figure 1.

The second method for determining salicylic acid elimination rate made use of a nonlinear anciysis. This method involved a computerized iterative technique of fitting blood level data asing a nonlinear least squares regression program. The least squares estimates of the kinetic parameters, and the standard deviations and confidence intervals of the parameters were all calculated.

The elimination rate constants as calculated by both methods are shown in Table 1. Statistical analysis using the student's t test at the 0.01 confidence level indicated there was no significant difference in elimination rate constants when calculated by either method. Further, no significant differences were found to exist in elimination Iate constants in animals treated with SA $110 \%$ salicylic 


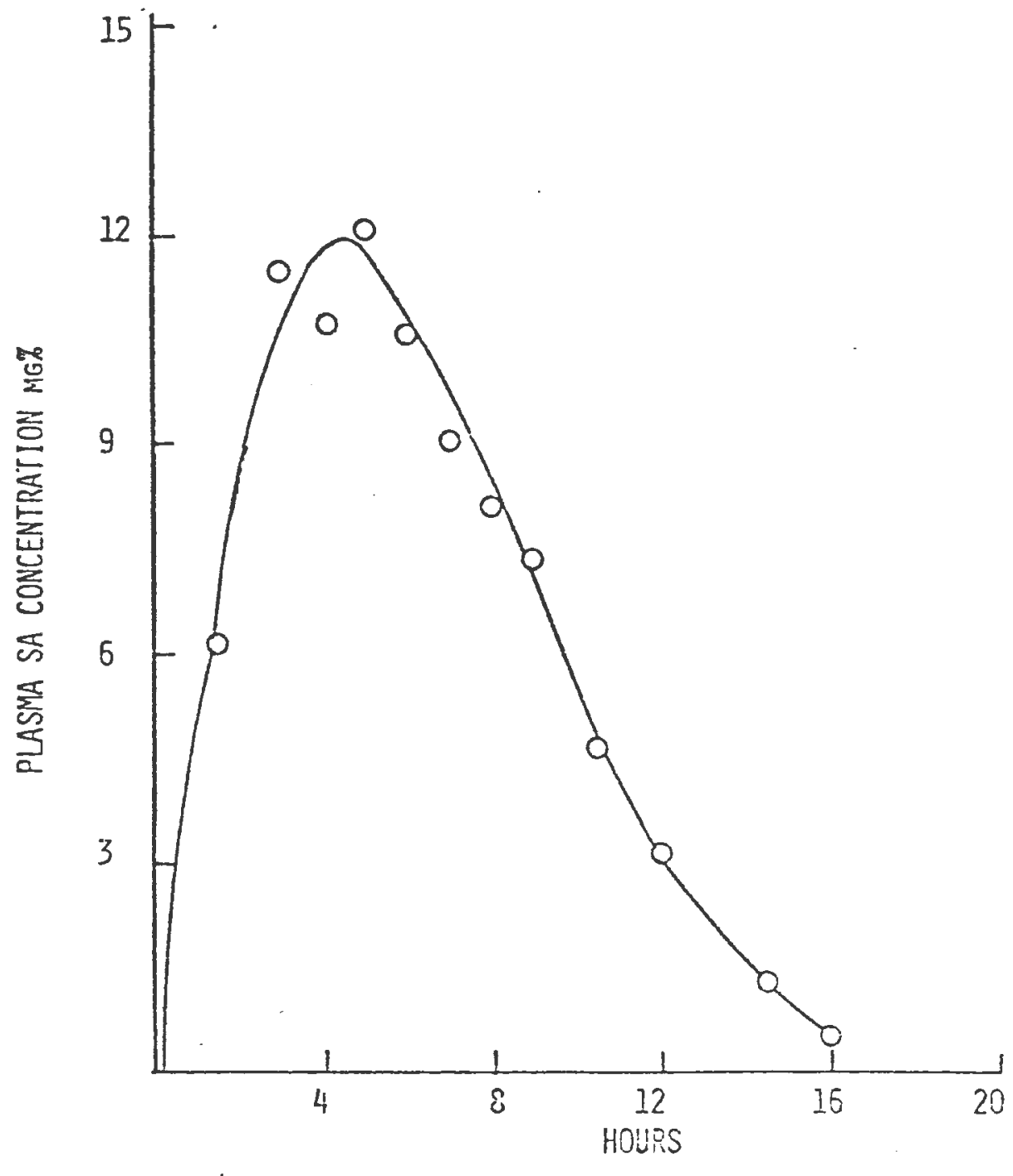

Figure 1 - Salicylic Acia concentration In The Eabbit Following the Tokical Application oE 10 : Salicylic Acid Ointaent 
TABLE I.

Elimination Rate Constants For Salicylic Acid

Calculated By Two Standara Methods

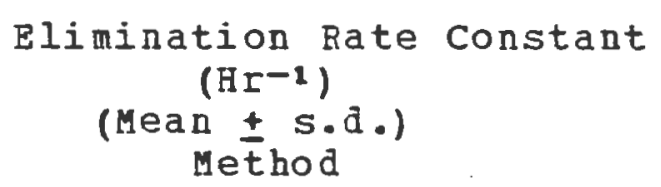

TPEATHENT

ELIUINATION PLOT

NONLIN

SA

8 Hours $\quad 0.409 \pm 0.27 \quad 0.289 \pm 0.07$

SA
4 Hours
$0.449 \pm 0.25$
$0.496 \pm 0.14$

$\mathrm{SAO}$

8 Hours

$0.272 \pm 0.02$

$0.269 \pm 0.07$ 
acid in hydrophilic ointment) for eight hours versus those treated with SA for four hours or those treated with SAJ (10\% salicylic acid and $10 \%$ urea in bydrophylic ointment) for eight hours. No differences in elimination rate constant were expected between treatments since the metabolism and excretion of salicylic acid is a function of the serum salicylic acid concentrations and would be expected to be independent of the type of ointment applied.

2. Half Life

Half lives for each treatment were calculated from the elimination rate constants determined by plotting the log salicylic acid concentration versus time and obtaining the slope of the elimination phase portion of the curve. Since salicylic acid has been shown (23) to follow first order kinetics at low doses, half-life was calculated according to equation $(6)$.

$$
\mathrm{T} 1 / 2=0.693 / \mathrm{ke}
$$

Equation (6)

Calculated half-lizes are presented in Table 2. No statistical differences were shown between any treatments. 
TABLE II.

Mean Salicylic Acid Half-Lives

TREATMENT
(mean $\pm S . \mathrm{d}_{\bullet}$ )

SA

8 Hours

$1.93 \pm 1.00$

$5 A$

4 Hours

$1.83 \pm 1.04$

$S A U$

8 Hours

$2.01 \pm 0.08$ 
3. Absorption Rate Constants

The Tagner-Nelson method is commonly used to characterize the absorption process. This technique is based on the one compartment open model, and can be used to obtain data from which the order of absorption and the appropriate rate constant can be calculated. The Wagner-Nelson equation is:

$$
A t / V=C t+k \int C p d t
$$

Equation (7)

Where;

At $/ \nabla$ is the amount of drug in the body at time $t$, divided by the volume of distribution $\nabla$, Ct represents the concentration of drug in the blood at time $T$, and the integral $\mathrm{k} \int \mathrm{cdt}$ represents the cumulative amount of arug eliminated to time $t$.

If the plot of the logarithm of the change in $a t / V$ $(d A t / V)$ versus $t$ ime is linear, the absorption process can be characterizeã as first order and an absorption rate constant determined.

The plots of the log of absorption rate versus time proved linear for all treatments; i.e., the absorption of topically applied salicylic acid can be characterized as a 
first order process. The slope of the line generated from data gathered from animals treated with SA for four hours, SA for eight hours, or SAU for eight hours yielded apparent first order absorption rate constants of $0.516 \mathrm{Hr}^{-1}, 0.340$ H ${ }^{-1}$, and $0.306 \mathrm{Hr}^{-1}$, respectively. Absorption rate constants for each treatment are shown in Table 3 and a $\mathrm{dat} / \mathrm{V}$ versus time plot is given in Figure 2.

Data obtained from the ragner-Nelson equation may be treated by a second method to calculate ka. At $t$ infinity. ct is equal to zero and the asymptotic value of the function is described as $A / V=k \int C p d$, where, $M / \nabla$ represents the total amount of drug absorbed divided by the rolume. By expressing the amount of drug absorbed at time $t$ as a percentage of the total amount absorbed (A/V) and subtracting from 100 , the percent remaining to be absorbed was calculated. It is critical that an accurate value of $\mathrm{A} / \mathrm{V}$ be used. Hagner has reviewed this method and discusses the causes of error in estimating $A / V$ and the effect this has on calculated absorption rate constants. Plotting the $\log$ of the percent remaining to be absorbed versus time yields an absorption plot from wich ka can be calculated by determining the slope of the line, Pigure 3. Absorption rate constants calculated using this technique appear in Table 3. Statistical analysis using the student's t test showed no significant difference $(\mathrm{p}=0.01)$ in the values of ka calculated by either the rate method of Wagner-Nelson or the percent remaining technique. The similarity of the 
TABLE III.

Salicylic Acid

Rate Constants

Calculated By Three Standard Methods

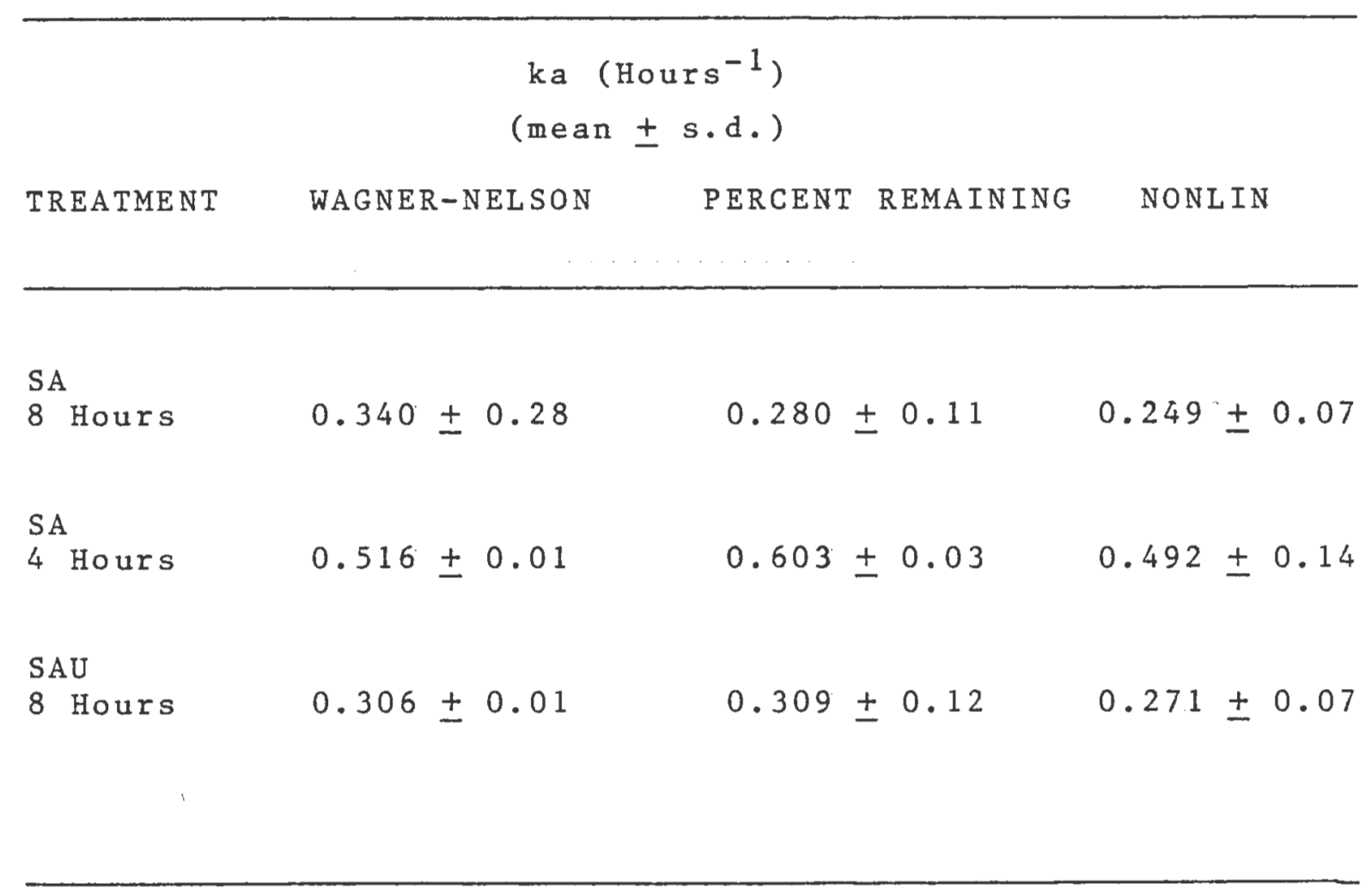




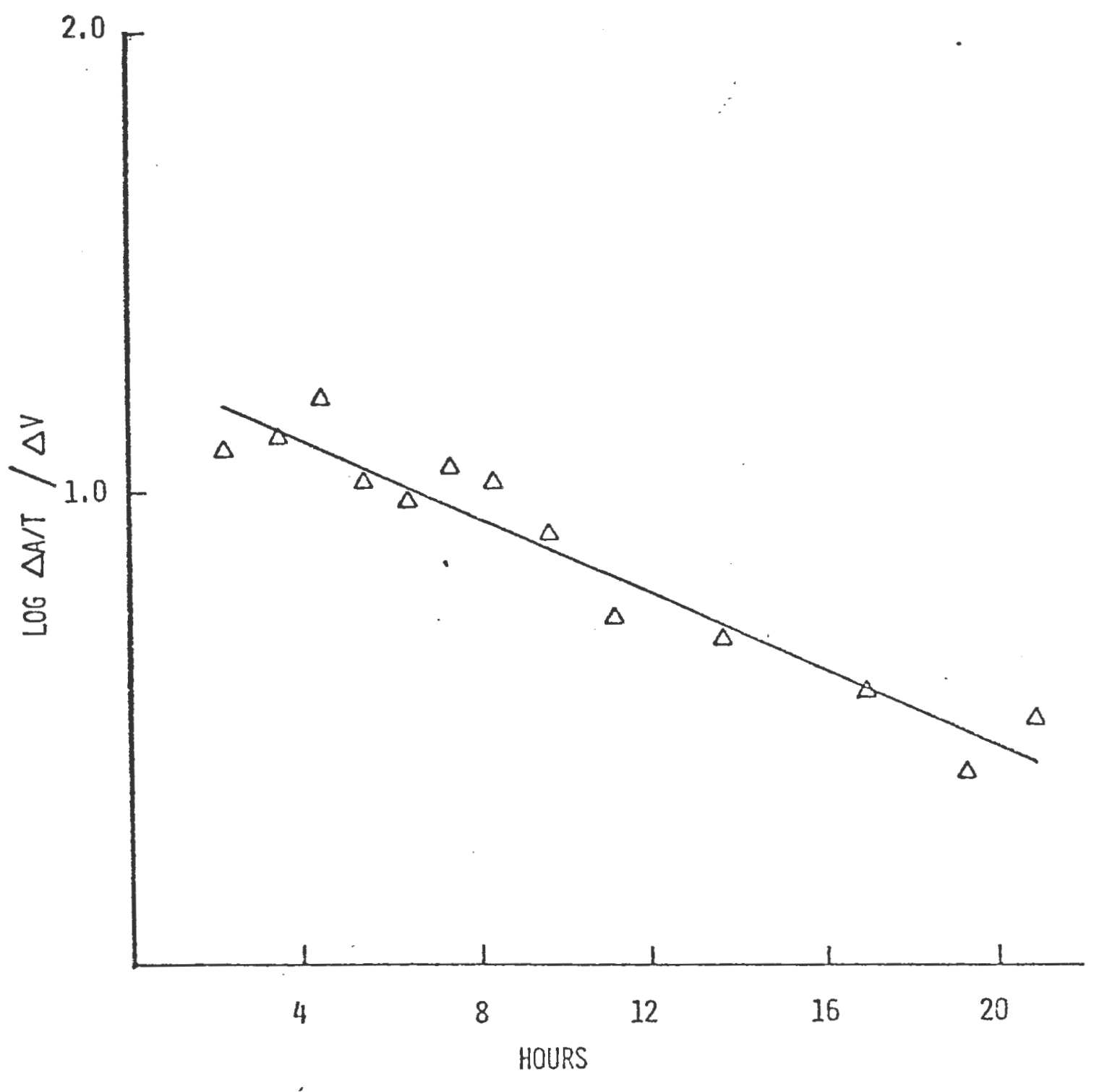

Figure 2 - Absorption Rate of Salicylic Acic Calculated By The hagner-Nelson Method. (Rabbit D) 


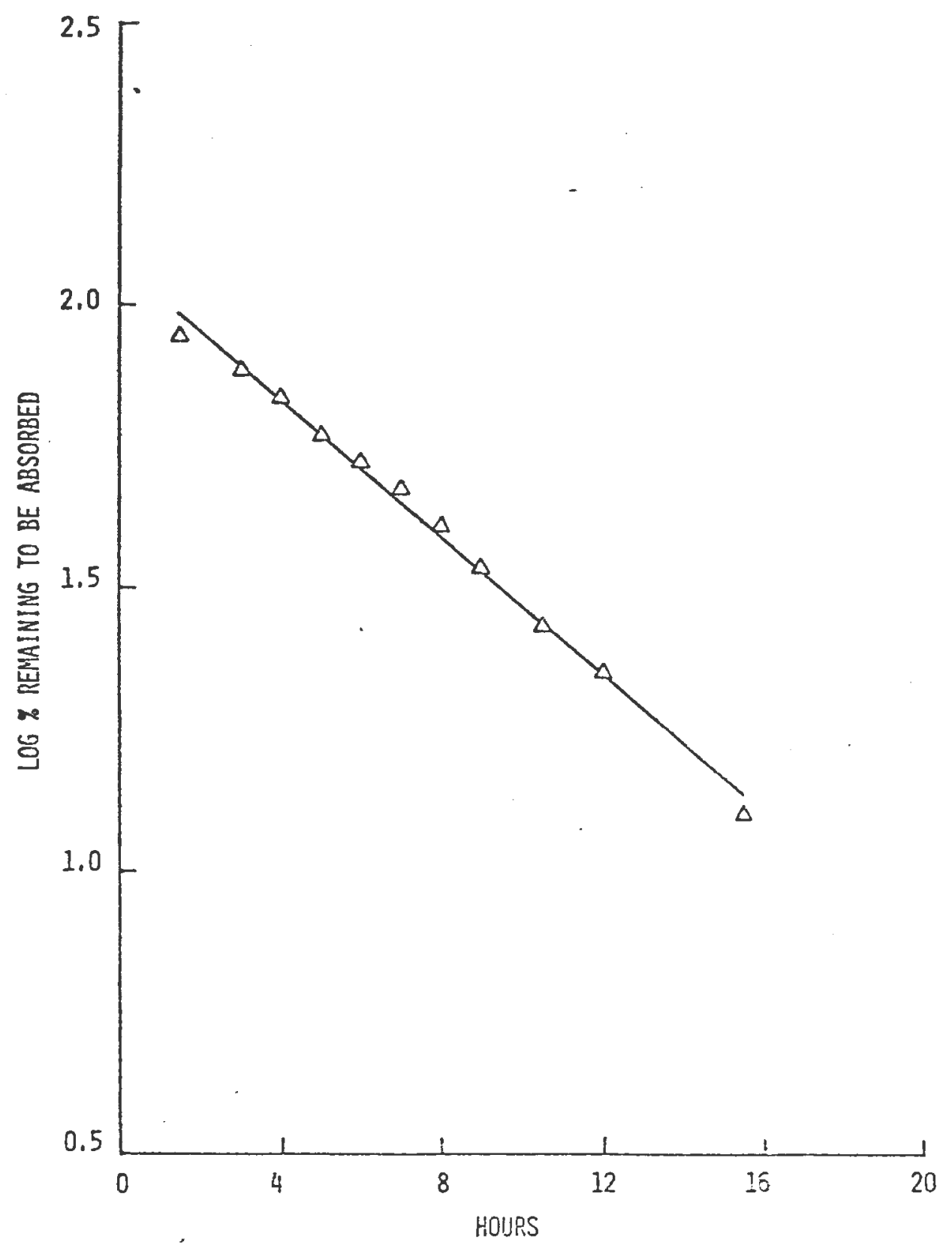

Pigure 3 - Percent Salicplic Acia Remaining To Be Absorbej (Rabbit E) 
absorption rate constants was evidence that the calculated value of $A / \nabla$ was accurate.

Since the absorption process was characterized as first order, fitting the one compartment open model, the data was treated by the method of nonlinear least squares regression (NONLIN), using the one compartment open model with a first order absorption rate constant, ka. Initial estimates of $k a$ and $k e$ were obtained from the ragner-Nelson and log concentration versus time plots respectively. The NONIN program uses an iterative process to "fit" a one compartment open model, with first order absorption to the blood level data. An example fit is shown in Figure 4. The absorption rate constants obtained by the NoNIIN fits appear in Table 3. At the 0.01 confidence level there was no significant difference between the values of ka calculated by this method and either of the methods previously discussed.

comparison of absorption rate constants obtained from animals treated with SA for eight hours with those treated with SA for four hours showed no significant difference at the 0.01 level. This result was expected since the absorption rate depends on the characteristics of the drug, its vehicle and the barrier properties of the skin and not the length of time of application.

Comparison of absorption rate constants calculated in animals treated wh SA ointment for eight hours with those treated Nith sAU cintment for eight hours showed no statistical difference. It appears that the adition of ten 


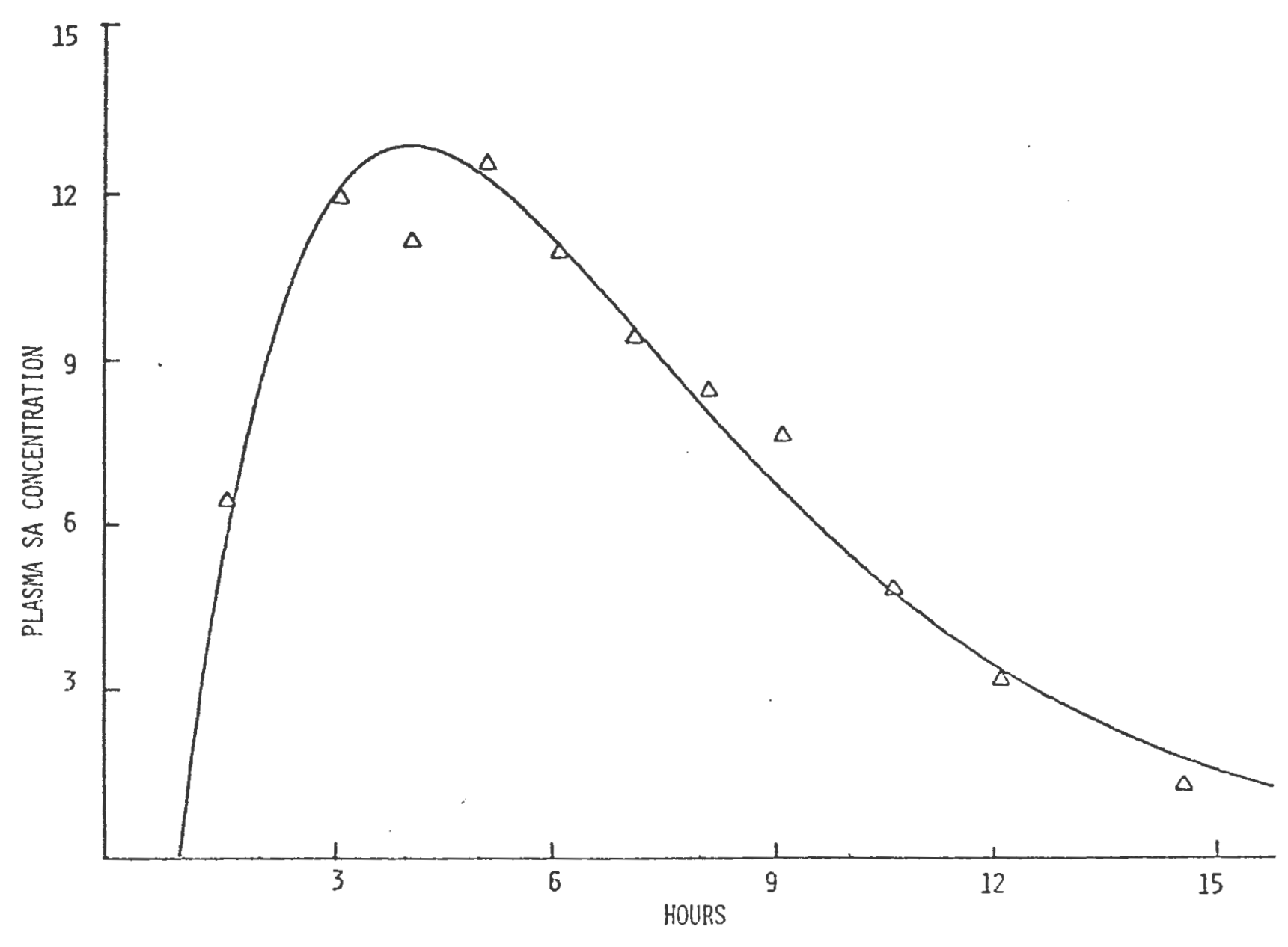

Figure 4 - Salicylic Acid Concentration Rabbit J (-) kepresents Computer Estimated Plasma Proiile $(\Delta)$ Are Experimental Data points 
percent urea to the "standard" ointment had no effect on the rate at wich salicylic acid entered the bloodstream. However, only a small number of animals were treated with the urea-containing ointment. As a result of the small number of degrees of freedom, the student's $t$ test would require a large difference in the values of $k a$ in order to show a significant difference between ointments. Comparison of the two ointment types among a larger population might show significant differences in the rate of absorption. However, it seems unlikely that the addition of ten percent urea would show any clinically significant increase in the absorption of topically applied salicylic acid.

\section{Lag Tine}

The lag time may be defined as the time required after application for the first order absorption process to to begin. Extrapolation of the line generated from the log percent remaining versus time curve to the $y$ axis will yield the estimated. lag time for each treatment. Calculated lag times are shown in Table 4.

Lag times calculated from animals treated for engit hours with SA ointment and those treated with SAO ointment vere significantly different at the 0.01 level. Similarly. significant differences were shown to exist for animals treated with SA ointment for eight hours compared with those treated with the same ointment for a four hour period. 


\section{TABLE IV.}

Salicylic Acid Absorption Lag Times

Calculated BY Two Standard Methods

\begin{tabular}{|c|c|c|c|}
\hline TREATMENT & $\begin{array}{l}\text { Lag Time } \\
\text { PERCENT }\end{array}$ & $\begin{array}{l}\text { (HOUIs) } \pm s \cdot d . \\
\text { REMAINING }\end{array}$ & NONLIN \\
\hline $\begin{array}{l}\text { SA } \\
8 \text { Hours }\end{array}$ & $1.09 \pm 0.16$ & $0.85 \pm$ & \pm 0.15 \\
\hline $\begin{array}{l}\text { SA } \\
4 \text { Hours }\end{array}$ & $0.86 \pm 036$ & 0.87 & \pm 0.02 \\
\hline $\begin{array}{l}\text { SAO } \\
8 \text { Hours }\end{array}$ & $0.99 \pm 0.13$ & $0.79=$ & \pm 0.08 \\
\hline
\end{tabular}


Differences in lag times vere unexpected and were most likely caused by inadequate sampling during the earliest stages of the absorption process; the earliest blood samples were not drawn until after the end of the calculated lag time. Lag times are generally useful for cases in yhich systemic absorption is desired, the significance in topical systems is of less general importance.

5. Bioavailability

One of the most important parameters for determining relative bioavailability is the area under the blood concentration time curve. Areas under the curve (AUC) can be calculated by the trapezoidal rule. This value is proportional to the amount of drug absorbed. A Fortran computer program was written to calculate the AOC for each treatment; values of AOC are given in Table 5.

The relative extent of absorption can be determined by comparison of AOC of two products. The cumulative areas under the curve obtained from animals treated with SA for four hours and those treated with sAO ointment for eight hours were each compared to the SA ointment (without urea) applied for eight hours. No significant difference $(p=0.01)$ could be shown between either treatment and the "standard" ointment.

Serum salicylic acid concentrations for animals treated for eight hours generally did not peak for five to six hours 
Table $\nabla$.

Areas Under The Curve For

Topically Applied Salicylic Acid In The Rabbit

Treatment $\quad$ (mg. ml. -1 Hours)

SA

8 Hours

$141.35 \pm 39.54$

SA

4 Hours

$$
28.95 \pm 66.52
$$

SAT

8 Hours

$154.30 \pm 10.04$ 
following application of the ointment, in agreement with published reports $(43,44)$. Those treated for only four hours were essentially still in the absorptive phase when the ointment was remored, therefore, less salicylic acid was available for absorption. The wide variation in AUC calculated from animals treated for eight hours and the small number of animals treated for four hours most likely made the paired $t$ statistic insensitive to differences in AdC's for these tro groups. Comparison of the area under the curve between larger populations may be necessary to show any significant difference between these treatments.

Although no significant difference in areas under the curve could be demonstrated in animals treated with SAU ointment compared with those treated with salicylic acid alone, it is not possible to conclude that there is no effect of ointment on AUC. Because blood level data were obtained from only two animals treated with urea, the test statistic must necessarily be very large in order to show significance. Further work using more animals would be necessary to reach any conclusion regarding the effect of urea on the absorption of salicylic acid measured as the area under the blood level time curve.

6. Feeding Studies

In contrast to previous studies $(43,44)$ in which animals were fasted throughout an experiment (i.e. for the 
period of ointment application and the entire period of blood sampling) a series of experiments was completed in which animals were fed during the sample collection period. Fifteen hours following the application of SA ointment, four animals vere fed approximately 30 grams of Charles River Rabbit Chow. A representative salicylic acid concentration time profile is shown in Figure 5. A second peak was observed in each profile within two or three hours following the feeding of the test animal. Since the second peak occurs approximately eight to ten hours after the remoral of the test ointment the peaks cannot be attributed to aditional absorption of salicylic acid through the skin.

The presence of the second peak can probably be attributed to biliary recycling. Biliary recycling can be defined as the process by wich drug is secreted into the bile and then passed into the small intestine. The drug can then be reabsorbed from the intestinal lumen into the systemic circulation. Rischel (60) has stated that recycling of drugs excreted into bile in unchanged form and for metabolites is possible, if they are either absorbed or modified by the gut flora, particularly after splitting of conjugates. The form of drug which is excreted into the bile also affects biliary recycling, drags wich form glucuronides have been shown to undergo recycling since the glucuronide can be readily cleaved to form the free drug in the bile. Salicylic acid is metabolized by the liver and 
its metabolites, SAG, SPG, GA, and SU, are found in the bile (25). If the unchanged drug and the glucuronides, sag and SPG account for the majority of the drug in the bile of rabbits, as in man, it is likely that biliary recycling is occuring.

The result of biliary recycling of drugs is commoniy seen as either a series of "irregularly spaced peaks" in the declining portion of a blood concentration time curve or simply a prolonged elimination phase. The characteristic single second peak shown in Figure 5 can be explained by the test conditions imposed in this experiment. The test animals were fed only a small quantity of food at a determined time, any food not consumed within thirty minutes was remored. Food is known to be a stimulus for the secretion of bile. Protein and fats in particular are known to increase bile flow (60). Since the rabbit is known to store bile and release it upon stimulation such as food (61). it is possible that the stimulus provided by the small amount of food caused the release of bile which in turn lead to the reabsorption of salicylic acid. 


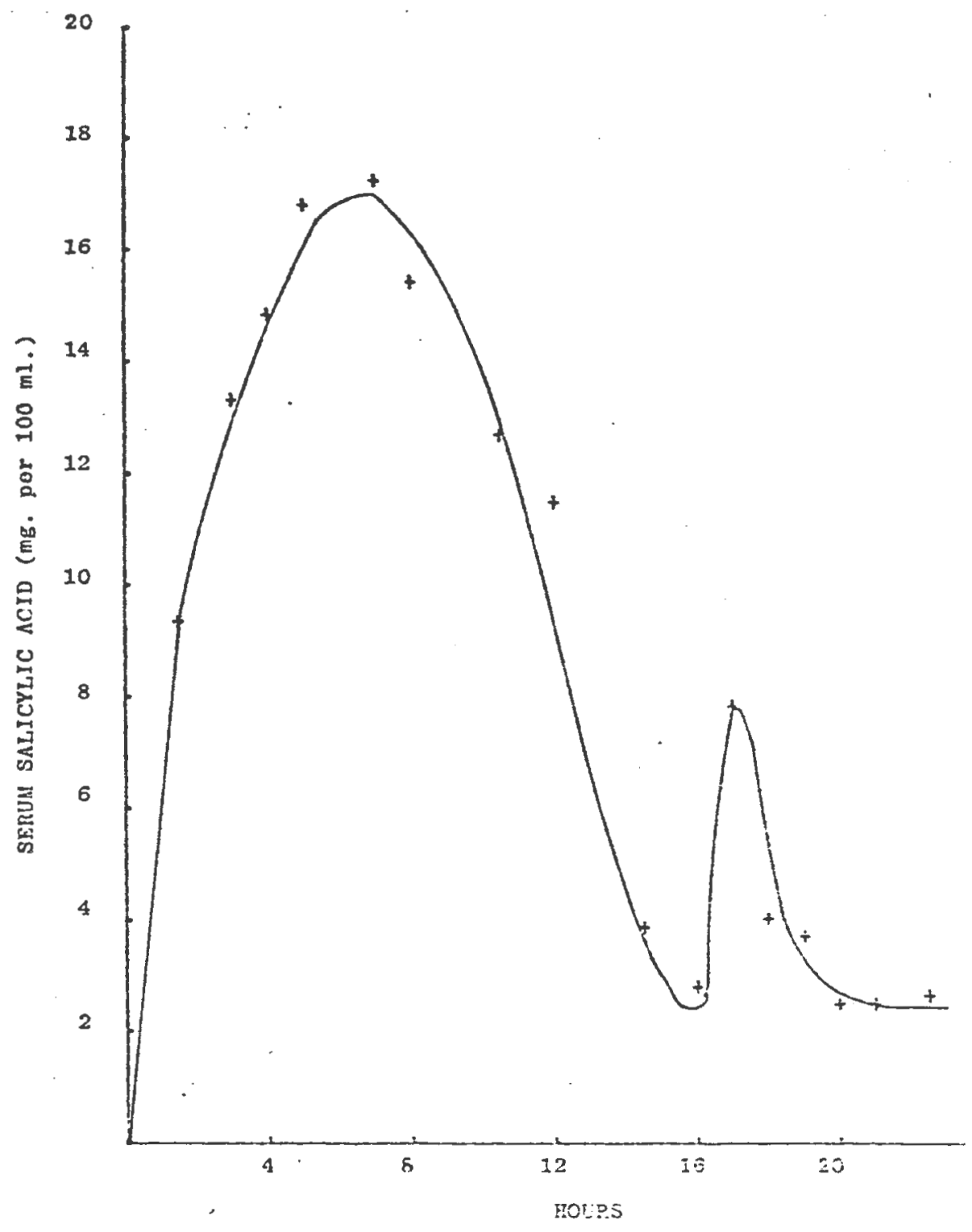

Figuire 5 - The Effect of Peesing on The Plasna Salicylic Acid Concentration Tiae Profile Following The Topical adiinistration of Salicylic acid. (Pabbit 3) 
B. Human Pharmacokinetics

1. $3 \%$ Salicylic Acid in $40 \%$ Polyethylene Glycol/nater solution

a. Intact skin

A mixture of polyethylene glycol 1500 and water was chosen as a vehicle for two reasons: first, as a Iiquid system, it was felt that diffusion through the vehicle would not be a rate-limiting step as is the case with some ointments, so that absorption rates obtained vould more accurately reflect the processes of diffusion into the skin; secondly, the polyethylene glycol 400/water mixture provided a system in which salicylic acid was sufficiently soluble to attain the concentrations required to match those of several clinically used products.

The systemic absorption of salicylic acid applied in the PEG/water vehicle for a period of two hours was minimal. plasma salicylic acid concentrations was found to be less than $1 \mathrm{mg} \%$ for each of two experiments.

The poor systemic absorption may be attributable to the formation of a glycol-salicylate complex resuliing in a molecule too large to easily pass the stratun 트므므므. Higuchi (28) has suggested the possibility of a complex formation between the higher molecular weight polyethylene glycols, e.g., PEG 6000 and salicylic acid. Several workers 
including colazzi (44) and stolar (43), have reported low serum salicylic acid concentrations when polyethylene glycol ointment was used. In adition, Nakano and patel (37) have demonstrated a low release rate of salicylic acid from this ointment. Salicylic acid absorption from the vehicle used in this investigation had not previiously been reported.

Although the systemic absoprtion of salicylic acia from this vehicle was limited (Figure 6) suggesting the possibility of complex formation in the lower weight glycols, the keratolytic effect was not. In both experiments, extensive karatolysis was observed within twenty-four hours of application of the test vehicle. The use of polyethylene glycols as vehicles for topically applied salicylic acid may prove to be useful in effecting keratolytic activity without the dangers of systemic absorption.

2. 10\% Salicylic acid in Hydrophylic Ointment

a. Intact skin

SA ointment' was applied, as previously described, to the forearm of four subjects. The skin at the site of application was intact, providing a condition of normal barrier function.

The systemic absorption of salicylic acid under these conditions was poor. Serum salicylic acid concentrations 


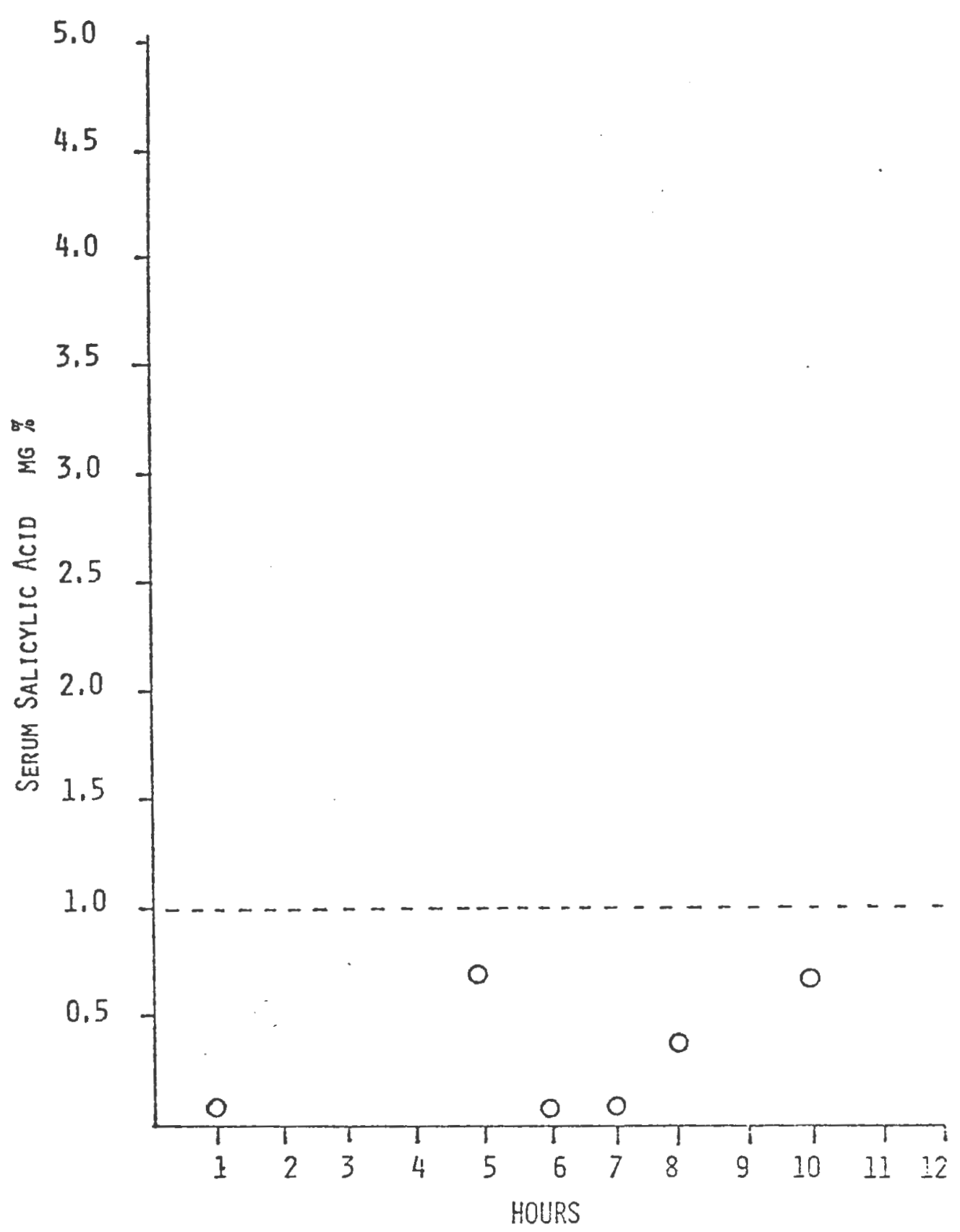

Figure 6 - Plasma Salicylic Acid concentration In Human Following The Percutaneous spplication of 3\% Salicylic Acid In 4O\% PEG/WATER. 
observed in each subject over an eight hour period were not significantly different from the serum concentrations taken prior to application of the test vehicle. These observations would seem to indicate that the possibility of systemic salicylic acid toxicity from the topical application of $10 \%$ salicylic acid ointment to intact skin is remote.

b. Stripped skin

The stratuan oㅡ묘묘 on the right forearm of two volunteers as disrupted as previously described. This technique was used in order to examine the absorption kinetics of salicylic acid ointment in a condition resembling that of diseased skin. sA ointment was applied for a period of three hours, under occlusion, after which time the ointment was removed by gently washing the test area with warm water and soap. Estimates of the kinetic parameters, i.e.. ka, ke, half-life, and lag time, derived from topically applied salicylic acid are therefore based on data collected from one subject due to difficulties in collection of samples from one of the other subjects. The parameters calculated by the methods previously descrioed are shown in Table 6.

The half-life calculated from the slope of the elimination phase of the log concentration versus time plot was 3.45 hours, in agreement with half-life values reported 


\section{TABLE $\nabla I$.}

Pharmacokinetic Parameters for Salicylic Acid obtained From The Percutaneous Application of Salicylic Acid In A Human Subject

\begin{tabular}{lc}
\hline PARAGTER & VALUE \\
Ka & $0.189 \mathrm{Hr} \cdot-1$ \\
Ke & $0.201 \mathrm{Hr} \cdot-1$ \\
Half-Life & $3.45 \mathrm{Hr}$. \\
Lag Time & $1.19 \mathrm{Hr}$. \\
\hline
\end{tabular}


for salicylic acid after low doses, i.e., when the elimination rate has been shown to be an apparent first order process.

Kinetic parameters obtained as previously described were used as initial estimates for NoNIIN. The computer generated parameters are shown in Table 6. The parameters obtained from NONIIN are statistically different from those obtained by the methods previously described. This is due to small number of data points obtained from the human subject which could not be properly fitted using the computer.

The absorption and elimination rate constants calculated from the administration of a single dose of topically applied salicylic acid can be fitted to a biexponential equation. This equation can later be used to predict blood levels resulting from multiple doses of the drug. Equation 8 is a common form of the biexponential equation used to predict plasina concentrations.

$$
C_{n}\left(t^{\prime}\right)=C_{0}\left(\frac{k}{k-K}\right)\left[\left(\frac{1-e^{-n k t}}{1-e^{-K} T}\right) e^{-K t^{\prime}}-\left(\frac{1-e^{-n K t}}{1-e^{k T}}\right) e^{-k t^{\prime}}\right]
$$

Equation

Where;

$t$ is the time measured from administration of the first dose

$t$ ' is the time measured from administration of the nth dose 
$\mathrm{n}$ is the dose number

Tis the dosing interval

$k$ is the first order absorption rate constant

$K$ is the first order elimination rate constant

Co is the initial drug concentration

Cn is the concentration following the nth dose.

Peak plasma salicylic acid levels attained following the three hour application of SA ointment to stripped skin in a human volunteer were approximately $8 \mathrm{mg} \%$ The entire arm is generally considered to represent nine percent of an individuals surface area (62). Therefore the area to which this ointment was applied, the forearm, represents approxinately five percent of the total surface area of the volun teer.

It is not uncommon for psoriatic patients to suffer lesions over at least thirty percent of their body (54). This represents a six fold increase in the area of skin exhibiting decreased barrier properties when compared to tie condition created in human volunteers in the previous study. Application of $S A$ ointment in the same manner as described for the normal human volunteers to the affected areas of psoriatics with thirty percent involvement would represent a six fold increase in the dose of salicylic acid applied. Equation 8 can be used to predict plasma salicylic acid 
concentrations in psoriatics applying SA ointment to involved skin totalling thirty percent of body surface area. Values of ke and ka used for this prediction are those obtained from the normal volunteer treated for three hours. The dose used was six times that used in the volunteer, corresponding to the six fold increase in the area of ointment application. The dosing interval, T, was every six hours. Figure 7 shows the predicted plasma salicylic acid versus time profile when the dosing regimen described above was carried out for forty eight hours. Steady state salicylic acid levels were attained after five doses and a concentration of approximately $60 \mathrm{mg} \%$ was predicted. This level is well above the lower level of toxicity, 20 to $30 \mathrm{mg}$. While the values of $k a$ and ke used for this model were those obtained from only one experiment, it is probable that these values approximate those which would be seen in psoriatic patients, therefore, the predicted plasma concentrations appear reasonable.

Although kinetic parameters have not been characterized in psoriatic patients, it is apparent from this work that patients treated chronically with salicylic acid containing ointments face the potential hazard of salicylate toxicity. 


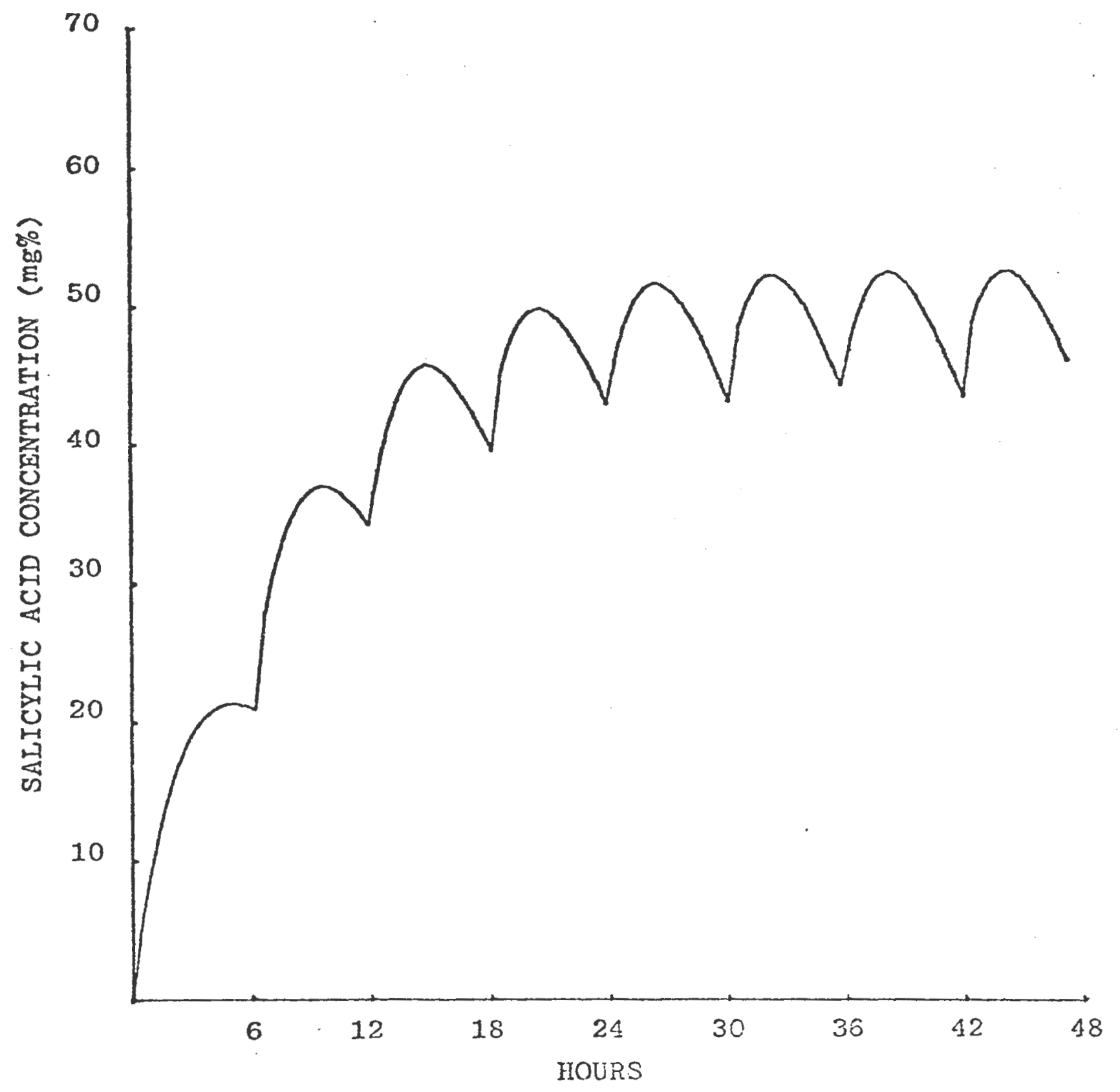

Figure 7 - Predicted Plasma Salicylic Acid Concentration In A patient hith $30 \%$ Involvement of Total Skin Surface Area. 


\section{CONCLUSIONS}

1. Absorption and elimination rate constants for topically applied salicylic acid were calculated in rabbits and humans. Values obtained were of the same magnitude in both species.

2. The addition of ten percent $w / w$ urea to hydrophylic ointment containing ten percent salicylic acid bad no significant effect on either the rate or extent of the systemic absorption of salicylic acid.

3. Absorption of salicylic acid from hydrophylic ointment appeared to be complete in approximately six hours after initial application of the test ointment.

4. Feeding of the test animals was found to cause a second peak in the plasma concentration versus time profile at a point approximately ten hours after the removal of the test ointment. The possibility of biliary recycling is discussed .

5. Application of three percent salicylic acid in a polyethylene glycol/water vehicle showed no detectable absorption of salicylic acid, although marked keratolytic effect yas observed.

6. The application of ten percent salicylic acid in hydrophylic ointment to the skin of human volunteers with "normal" skin resulted in no detectable blood levels of 
salicylic acid. The same ointment applied to volunteers whose skin had been stripped with adhesive tape resulted in plasma salicylic acid concentrations of approximately $8 \mathrm{mg}$ percent.

6. Application of the kinetic parameters calculatea from human volunteers to multiple dosing regimens demonstrated the possibility of toxic levels of salicylic acid when applied topically every six hours over a twenty-four hour period.

Future work

Several possibilities for further investigation have resulted from this work:

1. To examine the possibility of biliary recycling of salicylic acid. This would be conducted by examining the effect of food on the release of bile into the duodenum. The effect of preventing the secretion of bile into the duodenum." either by blocking the bile duct or cannulating the bladaer and altering the flow of bile away from the duodenum ill be studied.

2. To perform multiple dosing of topically applied salicylic acid to determine the accuracy of predicted plasma concentrations and to examine the potential for attaining toxic levels in the plasma.

3. To examine the keratolytic activity of salicylic acid in high molecular weight polyethylene glycol ointment bases 
in order to determine their therapeutic efficacy. If therapeutically effective, high molecular weight polyethylene glycols may provide a vehicle for topical preparations containing salicylic acid from which the systemic absorption would be minimal.

4. To characterize the pharmacokinetics of topically applied salicylic acid in the clinical setting, using psoriatic patients and in so doing to characterize tine rate and extent of absorption of salicylic acid as a function of severity of disease state and types of psoriatic lesions involved. 
VI. REFERENCES

1. C. J. Young, South. Med. J., 4느, 1075 (1952).

2. E. P. Cawley, N. T. Peterson, and C. E. Wheeler, JAMA, 151_ 372 (1952).

3. J. F. vonteiss, and T. F. Lever, Arch. Dermatol, 90. 614 (1964).

4. C. P. Iindsey, Med. J. Aust., 1, 353 (1968).

5. R. J. Feldman, and J. I. Mailbach, J. Invest. Dermatol., 50 , 351 (1968).

6. R. J. Scheuplein, "Properties of the Skin as a Uembrane". Chapter 10 of Pharmacology and the skin, vol. 12 of Advances in Biology of Skin Villiam Montagna, Eugene J. Vanscott, and Richerd B. Stoughton, Eds.. Meredith Corporation, Ney York, 1972, p. 125 - 151 .

7. Marvin Katz, "Design of Topical Drug Products: "Pharmaceutics", Chapter 4 of grug Design, vol. 4 , E.J Arienis. 'Bd.. of Medicinal Chemistry, A Series of lonographge George de Stevens, Ed.,

Academic Press, New York, 1973, p. 93 - 143.

8. K. C. James, Drug Dev. Com, 2, 453 (1976).

9. M. Ereeman, E. Alvarez, H. Draize, Fed. Proc., 9, $273(1950)$.

10. R. K. Loeffler, V. Thomas, in J. H. Hills, "percutaneous Abosiption", Chapter 12 of Pharmacology and the skin, vol. 12 of Advances in Biology of Skin, Wilizan Montagna, Eugene J. Vanscott, and Richard B. Stoughton, Eds., Meredith Corporation, New York, 1972 
p. $125-151$

11. A. Scott, op. cit., p. 172.

12. M. Katz, op. Cit., p. 101.

13. I. H. Blank, J. Invest, Dermatol, 21, 259 (1953).

14. R. J. Scheuplein, op. cit.e p. 134.

15. B. Idson, J. Pharm. Sci., 57, 1 (1968).

16. I. Brody, J. Oltrastruct. Res., 4, 264 (1960).

17. R. J. Scheuplein, opp.cit.. p. 139.

18. 1. Katz, op.cit.e p. 103.

19. R. J. Scheuplein, I. H. Blank, G. Brauner, and D. J. MacParlane, J. Invest. Dermatol., $\underline{52}_{2} 63$ (1969) .

20. L. S. Goodman, and A. Gilman, The Pharmacological Basis of Therapeutics, $4 \mathrm{th}$. Id., MacMillan Company, Hew York, 1970, Chapter 17.

21. I. S. Goodmar, and A. Gilman, op. cit.e p. 324.

22. J. F. Zarlonski, S. Karxztes-NagY, R. F. Mais and Y. T. Dester, Bioche日. J. 57, 301 (1954).

23. G. Lery and T. Matsuzawa, J. Pharm. Exp. Ther., 156, 285 (1967).

24. G. Levy, J. Pharm. Sci., 54, 959 (1965).

25. G. Levy, T. Tamchiro, and L. Amsel, 
Clin. Pharmacol. Ther., 13, 258 (1968).

26. A. J, Cummings, and B. K. Martin, J. Pharm. Sci., 5ㄱ. 891 (1968).

27. D. C. Heleod, and R. D. Lauper, Eds., "Jse of Salicylates in Rheumatoid arthritis", in The Clinical Pharmacy Revien, State University of New York at Buffalo, School of Pharmacy. Issue $6,1975$.

28. T. Higuchi, J. Soc. Cos. Chem., 11, 85 (1960).

29. B. Poulsen, "Design of Topical Drug Products: Biopharmaceutics", Chapter 5, of Druq Desiqn, vol. 4, E. J. Ariens, Ed., of Medicinal Chemistry. A Series of Monographs, George De Stevens Ed., Academic Press, New York, 1973, p. 155.

30. E. J, Lien, and G. L. Tong, J. Soc. Cos. Chem. 24. 371 (1973).

31. H. Barr, J. Pharm. Sci., 51, 395 (1962).

32. B. Idson, J. Pharm. Sci., 64 901 (1975)..

33 G. Kimura, orient. J. Dis. Inf. 2ㅇ․ 15 (1940), in Marr, loc. cit.e p. 395.

34. H. Nogani, J. Hasegawa, M. Hanano, Bharm. Buli. Tokyo, $4,347(1956)$, in 1. Barr, 10․ㅡㄹㅗ. p. 395 .

35. J. Howze, and N. Billups, Am. J. Pharm., $\underline{3}_{2}$ 193 (1966).

36. N. Billups, and N. K. Patel, Am. J. Pharm. Bd., 34. 190 (1970).

37. H. Nakano, and N. K. Patel, J. Pharm. Sci., 59, 
$985(1970)$

38. T. Higuchi, and J. Lach, J. Am. Pharm. Assoc., Sci. Ed., $4 \underline{3}_{2} 465$ (1954).

39. C. W. Whitworth, J. Pharm. Sci., 57, 1540 (1968).

40. F. Bottari, J. Pharm. Sci., 63, 1779 (1974).

41. T. Higuchi, and I. Kozumi, J. Pharm. Sci., 50. 200 (1961).

42. H. Strakosch, Arch. Dermatol. Syphilo1., 4I, 16 (1943), in M. Barr, 1oc. cit.e p. 395.

43. M. Stolar, G. Rossi, and M. Barr, J. Am. Pharm. Assoc., 49, 144 (1960).

44. J. I. Colazzi, Am. J. Fharm. Ed., 34, 185, $(1970)$.

45. J. Stelzer, J. Colazzi, and P. Wordack, J. Pharm. sci, 57,1732 (1968).

46. J. Shelmire, Arch, Dermatol, 82,24 (1960).

47. M. Mashitake, T. Anmo, I. Tanaka, T. Arita, M. Nakano, J.. Pharm. Sci., 64, 397 (1975).

48. D. Wurster, and S. Kraemer, J. Pharm. Sci., $\underline{5}_{2}$ 288 (1961).

49. T. Arita, R. Hori, T. Anmo, H. Fashitake. H. Akatsu, and T. Yajima, Chem. Pharm. Bull., 18. 1045 (1970).

50. F. Marcus, J. Colazzi, H. Barry III., J. Pharm Sci., 59, 1616 (1970). 
51. M. Shen, A. Danti, and F. Bruscato, J. Pharm. Sci. 65 , 1780 (1976).

52. Dale E. Furster, Some Practical Applications of Percutaneous Absorption Theor $y^{\prime \prime}$. Chapter 11 of, Pharmacology and the Skinh, vol. 12 of, Advances in Biology of Skin, Hilliam Montagna, Eugene J. Vanscott, and Richard B. Stoughton, Eds., Meredith Corporation, New York, 1972, p. $153-168$.

53. H. Hashitake, T. Yajima, T. Anmo, T. Arita, and R. Hori, Chem. Pharm. Bull., 21, 2444 (1973).

54. R. Taylor, and K. Halprin, Arch. Dermatol. 111, $740(1975)$.

55. L. S. Goodman, and A. Gilman, op. cit.e p. 324

56. M. Clay, and J. Nelson, J. Pharm. Sci., 43, $230(1954)$.

57. A. Saltzman, J. Biol. Chem., 174, 394 (1948).

58. D. Trinder, Biochem. j. $\underline{301}$ (1954).

59. M. Biber, and C. T. Rhodes, Clin. Chimica. Acta., 54, 135 (1974).

60. M. A. Ritsche1, Handbook of basic Pharmacokinetics, Drug Intelligence Publications, Inc., Hamilton, Illinois, 1976, p. 191.

61. . J. Jusko, and G. Levy, J. Pharm. Sci., 56, $58(1967)$.

62. L. S. Brunner, D. S. Suddarth, B. B. Faries, K. A. Galligan, D. B. Lavoie,

A. C. Schwalenstoceker, The Lippincott Kanㅡ므므 of Hursing practice, J. B. Iippincott Company Philadelphia, 1974 p. 602 . 


\author{
Certification of Review \\ and \\ Special Implementation of Institutional Assurance
}

The proposal titled "Study of Percutaneous Absorption of Salicylic Acid" submitted on behalf of Bruce K. Birmingham, J.W. Cooper - 1/5/76 has been reviewed by the assembled members of the IRB whose signatures below appear in accordance with the requirements of the DHEW regulation of Protection of Human Subjects (45 CFR 46\%).

(1) Wis IRB has determined that the subjects in this activity are at risk. The risks are: local skin irratation, Tinnitus and the possibility of infectlon due to blood puncture.

(2) This IRB has determined to its satisfaction that the following safeguards against the specified risks are adequate:

All subjects will be carefully screened before testing for broken skin or history of blood a: vilation disorders, ulcers, or aspirin sensitivity. The tests wi.h. os conducted in the University (Potter) Infirmary under the direction of an M.D., blood samples will be taken by a licensed medical laboratory technologist, utilizing the best antiseptic techniques.

(3) This IRB has determined to its satisfaction that the risks to the subjects are so outweighed the sum of the benefits to the subject and the importance of the krotiledge to be gained as to warrant a decision to allow the subject to accept the risks. This determination is based upon the following benefits or reasons:

The risks involved are small since toxic hazard is both unlikely and of minor nature.

(4) This IRB has determined to its satisfaction that legally effective informed consent is to obtalned and properly documented in accordance with the requirements specifled in the regulation ( 45 CFR 46.3 (c) and 46.10 ). The informational statement to be given or read to each prospective subject before his participation in the activity is attached.

(5) This InB agrees to arrange for the continuing exchange of infornation and advice between itself and the activity director on any matters affecting the rights and welfare of human subjects who participate in the activity. The specific instructions, advice, counsel and the conditions imposed by the IRB for the conduct of the activity are: 
The next scheduled meeting of the IRB for review of this activity is December 1976. The IRB may be called into an interim review session by the Chairman at the request of any member, an institutional official or the project director to consider any matter concerned with the rights and welfare of any subject.

(6) This IRB has determined to its satisfaction that this institution will have avallable the professional attention and facilities that may be needed for subjects who may suffer physical, psychological or other injury as a result of participation in the activity.

$$
\text { C.Tinim }
$$

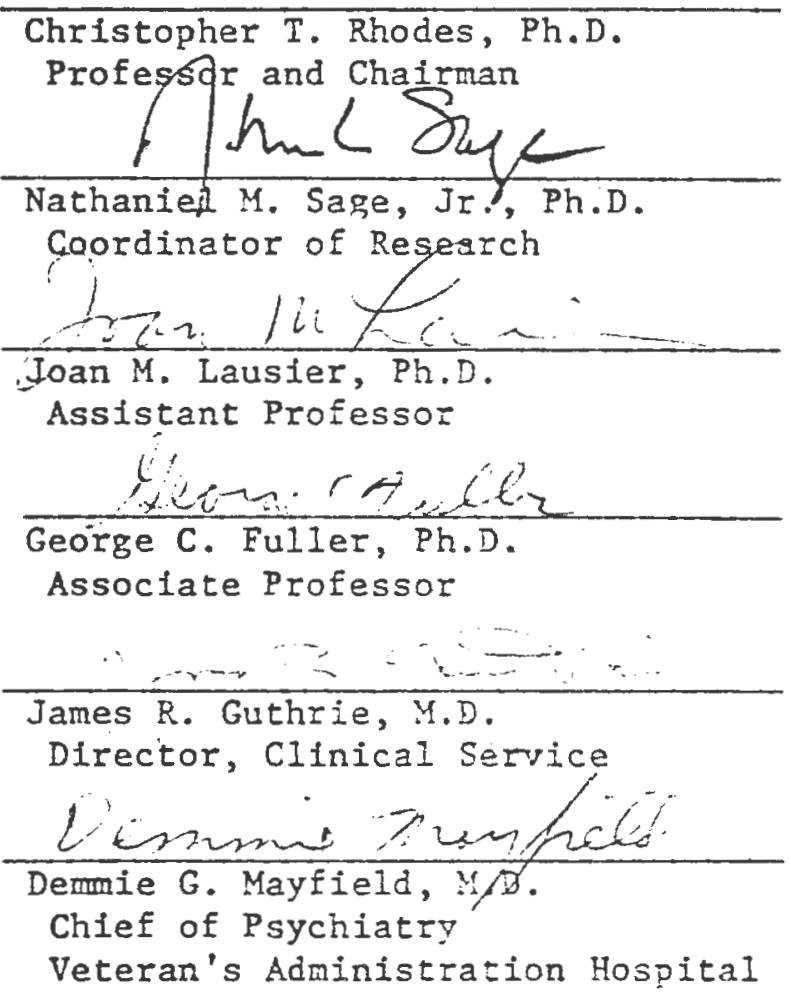


Study of the Percutaneous Absorption of Salicylic Acid, College of Pharmacy

University of Rhode Island 1976

Investigators:

Bruce K. Birmingham, B.S.

Graduate Student

James W. Cooper, B.S., Ph.D.

Assistant Professor and Director

of Clinical Pharmacy

Christopher T. Rhodes, B.Pharm., Ph.D.

Professor and Chairman, Department of Pharmacy

Objectives: $\quad$ To examine and characterize the pharmacokinetics of salicylic acid absorption through the skin, in particular to define the rate and extent of drug absorption. An attempt to characterize interpatient variability will also be undertaken.

Preliminary Screening of Subjects

All those being considered for involvement in this study will be healthy volunteers between the ages of twenty and fifty, with no broken skin or history of blood coagulation disorders, ulcers or aspirin sensitivity. In addition, any other medical abnormality which in the opinion of the medical consultant or investigators is likely to complicate the study will result in exclusion from the study. All subjects will be carefully interviewed and pertinent medical information gathered. All participants will be provided the opportunity of reading and having carefully explained the Informed Consent Form. In addition, participants will be provided the opportunity for further questioning, of the investigators. Any subjects may withdraw from the study at any time during, the investigation. Participants will be required to abstain from aspirin containing products for one week prior to the test period and all drugs and alcohol for 43 hours prior to the test period. 
A one week "wash-out" period will be allowed between tests.

The tests will be conducted in the University (Potter) Infirmary under the direction of an M.D., blood samples will be taken by a licensed medical laboratory technologist.

Salicylic acid will be supplied in the form of a buffered aqueous solution in which the subject will soak his arm for a period of two hours. Blood samples, to be drawn by a licensed medical laboratory technologist, will be taken immediately preceeding immersion in the test solution and at the following times from the time of inversion, 0.5 hours, $1.0,1.5,2.0,4.0$, and 3.0 hours. The test will require a $10 \mathrm{ml}$ sample to be taken at each interval, for a total of $70 \mathrm{mls}$ of blood from each volunteer for each test. Participants will also be asked to provide a urine sample twenty-four hours following application of the test solution.

Minimum toxic levels of salicylic acid have been reported at between 20 and $40 \mathrm{mg} \%$. The current study is designed so that serum salicylate levels do not exceed $10 \mathrm{mg} \%$. 
U.R.I. 1976

Investigators: Bruce K. Birmingham, B.S.

James W. Cooper, B.S., Ph.D.

Assistant Professor and Director of Clinical Pharmacy

Christopher T. Rhodes, Ph.D.

Professor and Chairman of Pharmacy

Salicylic acid is a drug used in the treatment of several types of skin disorders. It is normally supplied to the patient in the form of an ointment or cream which is applied topically (i.e., directly to the affected areas of the skin.)

The objective of this research project is to study the absorption characteristics of salicylic acid when applied to normal volunteers in the form of an aqueous (water) solution.

Should you volunteer to participate in this project you will be required to abstain from all drugs and alcohol for a period of 48 hours prior to each test, as well as abstaining from all aspirin containing compounds for one week prior to the beginning of the test period and for the duration of the study, approximately ten weeks. The test solution will be prepared by the investigators and will contain $3 \%$ salicylic acid in water. You will be asked to place your arm in a bath containing the test solution for a period of two hours. Blood samples will be taken during the course of topical application and at four and eight hours after the start of the test. You will be asked to supply urine samples for a period of 24 hours following each treatment.

The possible effect of the salicylic acid test solution application is local skin irritation at the site of application of the test solution. Tinnitus (i.e., a ringing sensation in the ears) is the first sign of toxicity which rarely occurs below salicylate levels of $20 \mathrm{mg} \%$. This study is designed not to exceed levels of $10 \mathrm{mg} \%$.

Should you volunteer for this project you will be free to withdraw from participation at any time during the investigation. You will not be identified in any publication resulting from this work.

I, having carefully read and understood the above and having been given the opportunity of asking questions regarding the above project now give my informed consent to participation in the Study of the Percutaneous Absorption of Salicylic Acld, University of Rhode Island.

Date

Witness 